\title{
Schemes with well-controlled dissipation. Hyperbolic systems in nonconservative form
}

\author{
Abdelaziz Beljadid ${ }^{1}$, Philippe G. LeFloch ${ }^{2}$, \\ Siddhartha Mishra ${ }^{3}$, and Carlos Parés ${ }^{4}$
}

\begin{abstract}
We propose here a class of numerical schemes for the approximation of weak solutions to nonlinear hyperbolic systems in nonconservative form - the notion of solution being understood in the sense of Dal Maso, LeFloch, and Murat (DLM). The proposed numerical method falls within LeFloch-Mishra's framework of schemes with well-controlled dissipation (WCD), recently introduced for dealing with small-scale dependent shocks. We design WCD schemes which are consistent with a given nonconservative system at arbitrarily high-order and then analyze their linear stability. We then investigate several nonconservative hyperbolic models arising in complex fluid dynamics, and we numerically demonstrate the convergence of our schemes toward physically meaningful weak solutions.
\end{abstract}

\section{Introduction}

This is a follow-up to the papers [17, 32], in which two of the authors have introduced a class of schemes, refered to as the schemes with well-controlled dissipation (WCD), which allow one to compute, with robustness and accuracy, small-scale dependent shock wave solutions to nonlinear hyperbolic systems of conservation laws. We do not attempt to review the problem of nonclassical shocks and the corresponding numerical techniques and, instead, we refer to the textbook by LeFloch [29] together with the recent review by LeFloch and Mishra [32] and references therein.

In the present paper, we investigate nonlinear hyperbolic models in nonconservative form, such as those arising in the modeling of two-phase (liquid-vapor) flows and shallow water (two-layer) flows. Shock waves appear often in these contexts and developing reliable numerical methods is an essential challenge. Specifically, we consider nonlinear hyperbolic systems in one spatial variable, of the general form

$$
\begin{aligned}
& U_{t}+A(U) U_{x}=0, \quad U=U(t, x) \in \mathbb{R}^{N}, \\
& U(0, x)=U_{0}(x),
\end{aligned}
$$

where $U$ is the vector of primitive unknowns and $A=A(U)$ is a smooth map defined on (a subset of) $\mathbb{R}^{N}$. We assume that, for each relevant $U$, the matrix $A(U)$ admits distinct real eigenvalues denoted by $\lambda_{1}<\lambda_{2}<\ldots<\lambda_{N}$ and, therefore, a corresponding basis of right-eigenvectors, denoted by $r_{j}(U)_{1 \leq j \leqslant N}$. We are interested in (possibly) discontinuous solutions to (1.1) that can be realized as (singular) limits of (smooth) solutions to systems with vanishing diffusion and (possibly) dispersion, that is,

$$
\begin{aligned}
& U_{t}+A(U) U_{x}=\epsilon\left(B(U) U_{x}\right)_{x}+\alpha \epsilon^{2}\left(C_{1}(U)\left(C_{2}(U) U_{x}\right)_{x}\right)_{x}, \\
& U(0, x)=U_{0}(x) .
\end{aligned}
$$

\footnotetext{
${ }^{1}$ Department of Civil and Environmental Engineering, Massachusetts Institute of Technology, 77 Massachusetts Avenue, Cambridge, Massachusetts 02139, USA. Email: beljadid@mit.edu

${ }^{2}$ Laboratoire Jacques-Louis Lions \& Centre National de la Recherche Scientifique, Université Pierre et Marie Curie, 4 Place Jussieu, 75252 Paris, France. Email: contact@philippelefloch.org.

${ }^{3}$ Seminar for Applied Mathematics (SAM), ETH Zurich, Rämistrasse-101, Zürich, 8092, Switzerland. Email: siddhartha.mishra@sam.math.ethz.ch.

${ }^{4}$ Departamento de Análisis Matemaático, Universidad de Maálaga, 29071 Málaga, Spain. Email: pares@uma.es.
} 
The challenging question of defining weak solutions to (1.1) was solved around 1990 by LeFloch and his collaborators. Recall that it was first proposed in [25] to rely on Volpert's product and in [26] to rely on traveling wave profiles associated with an augmented model taking into account small-scale effects. A general notion of nonconservative product was then introduced by Dal Maso, LeFloch and Murat (in the preprint included in [27], later published in [15]) who also solved the Riemann problem for nonconservative systems. Recall also that the existence of weak solutions in the DLM sense to the Cauchy problem was established for nonlinear hyperbolic systems (1.1) by LeFloch and Liu [31] via Glimm's random choice method when the initial data has small total variation. The existence of traveling wave solutions to nonconservative systems was established later on in [38]; see also [2].

The numerical investigation of nonconservative hyperbolic problems was initiated in Hou and LeFloch [22], motivated by an earlier study by Karni [24] for the system of gas dynamics equations. The class of nonconservative systems and, more generally, systems admitting small-scale dependent shock waves, leads to particularly challenging problems as was first studied by LeFloch and collaborators $[22,21,23,34,14,33]$ and later further investigated in $[8,37,1]$. Recall that a variety of approaches for nonconservative systems were proposed in the literature, especially by Gosse [20], Berthon and Coquel [3, 5, 6] together with LeFloch [7], Parés and collaborators [11, 12, 35, 36], Mishra and collaborators [9, 19], and Berthon, Boutin, and Turpault [4].

However, as pointed out in the review [32], robust and accurate schemes that can compute small-scale dependent shocks to nonconservative hyperbolic systems, especially with arbitrary large amplitude, still remain unavailable. It is precisely our objective in the present paper, as mentioned earlier, to built upon the WCD framework recently proposed by LeFloch and Mishra $[17,32]$ and to design schemes with well-controlled dissipation adapted to (1.2).

Recall that, in [17], the authors designed WCD schemes for the approximation of nonclassical shock waves to nonconvex (but conservative) hyperbolic systems. The extension of these schemes to nonconservative hyperbolic systems is addressed here. Throughout, we rely on the notion of the equivalent equation associated with a scheme, whose importance to handle small-scale dependent solutions was discovered by LeFloch and collaborators [22, 21, 34, 33]. The equivalent equation associated to a numerical method, which is a high-order PDE system obtained by a formal Taylor expansion, was also used to design numerical methods in [24] and [9]. The general idea is to design numerical methods such that the viscous terms in the equivalent equation coincides with the one arising from the physics. Although this methodology leads to numerical solutions that are closer to the correct weak solutions, convergence as $\Delta x$ tends to 0 cannot be expected in general, since the higher-order terms in the equivalent equation are not controlled [22].

As stated in (1.2), we take into account a vanishing diffusion and (possibly) a vanishing dispersion and our finite difference method, whose stencil consists of $2 p+1$ centered points, is applied in such a way that the diffusion and dispersion terms in the equivalent equation are of order $O(\Delta x)$ and $O\left(\Delta x^{2}\right)$, respectively, while the order of the higher-order terms is $O\left(\Delta x^{2 p}\right)$ at least. Importantly and this is one of the main features, our numerical method is provided with a parameter $c$ whose values is computed so that, given a certain tolerance $\tau$, the ratio between these higher-order terms and the physically meaningful ones at a shock is bounded by $\tau$. The numerical solutions therefore are expected to converge to the physically-meaningful weak solutions, when both $\Delta x$ tends to 0 and $p$ tends to $\infty$ (in agreement with the conclusion in [33] for nonclassical shocks).

We emphasize that the main goal of the numerical methods introduced here is the convergence to the correct weak solutions: they are not designed to compete in efficiency or shock-capturing properties with standard high-order numerical methods, which are relevant for a different class of problems, i.e. shocks that are insensitive to small-scale mechanisms.

An outline of this paper is as follows. In Section 2, we begin with a selection of nonlinear hyperbolic models in nonconservative form of particular interest in the dynamics of complex fluids. In Section 3, we introduce our class of schemes with well-controlled dissipation and we discuss various aspects of these schemes, including the important issue of establishing bounds on the time-step. Section 4 is then devoted to present numerical tests for the coupled Burgers system, the coupled cubic equations, a modified shallow water model, and, finally, the two-layer shallow water model. We conclude this paper with several remarks in Section 5. 


\section{A selection of nonlinear hyperbolic models in nonconservative form}

\subsection{The coupled Burgers equations}

In this section, we present some examples of nonconservative nonlinear hyperbolic systems. First of all, in [11] the authors proposed the following model problem for nonconservative hyperbolic systems:

$$
\begin{gathered}
\partial_{t} u+u \partial_{x}(u+v)=0 \\
\partial_{t} v+v \partial_{x}(u+v)=0 .
\end{gathered}
$$

The system can be rewritten in the form (1.1) with

$$
U=\left[\begin{array}{l}
u \\
v
\end{array}\right], \quad A(U)=\left[\begin{array}{ll}
u & u \\
v & v
\end{array}\right] .
$$

If the component equations of this system are added, then the Burgers equation for $w:=u+v$ is obtained:

$$
\partial_{t} w+\partial_{x}\left(\frac{w^{2}}{2}\right)=0 .
$$

Therefore, the equation (2.1) is termed the coupled Burgers system. The Burgers equation satisfied by the sum $w=u+v$ suggests the following entropy pair:

$$
\eta(U)=\frac{w^{2}}{2}, \quad q(U)=\frac{w^{3}}{3} .
$$

In order to set unambiguously the jump conditions across a shock, the small scale effects have to be taken into account. Following Berthon [3] we consider the vanishing diffusion term given by

$$
\begin{gathered}
\partial_{t} u+u \partial_{x}(u+v)=\epsilon_{1} \partial_{x x}^{2}(u+v), \\
\partial_{t} v+v \partial_{x}(u+v)=\epsilon_{2} \partial_{x x}^{2}(u+v) .
\end{gathered}
$$

In [3] the exact viscous profiles of the regularized system have been computed. In the limit $\epsilon_{1}, \epsilon_{2} \rightarrow 0$ this gives the correct (physically relevant) entropy solution of the Riemann problem for the coupled Burgers equation. This solution depends on the ratio $\epsilon_{1} / \epsilon_{2}$ : see [3].

Equations (2.1) can be rewritten in the following form

$$
\begin{aligned}
\partial_{t} w+w \partial_{x} w & =0, \\
\partial_{t} u+u \partial_{x} w & =0 .
\end{aligned}
$$

The system can be rewritten in the form (1.1) with

$$
U=\left[\begin{array}{l}
w \\
u
\end{array}\right], \quad A(U)=\left[\begin{array}{ll}
w & 0 \\
u & 0
\end{array}\right] .
$$

The eigenvalues of $A(U)$ are $\lambda_{1}(U)=w$ and $\lambda_{2}(U)=0$ and the corresponding eigenvectors

$$
r_{1}(U)=\left[\begin{array}{l}
w \\
u
\end{array}\right], \quad r_{2}(U)=\left[\begin{array}{l}
0 \\
1
\end{array}\right] .
$$

Therefore, the system is strictly hyperbolic if $w \neq 0$. Note that the first equation in (2.3) is decoupled from the second equation of this system and can be rewritten in conservative form. 


\subsection{The coupled cubic equations}

We consider next the system

$$
\begin{aligned}
& \partial_{t} u+2(u+v)^{2} u_{x}+(u+v)^{2} v_{x}=0 \\
& \partial_{t} v+(u+v)^{2} u_{x}+2(u+v)^{2} v_{x}=0 .
\end{aligned}
$$

The system can be rewritten in the form (1.1) with

$$
U=\left[\begin{array}{l}
u \\
v
\end{array}\right], \quad A(U)=\left[\begin{array}{cc}
2(u+v)^{2} & (u+v)^{2} \\
(u+v)^{2} & 2(u+v)^{2}
\end{array}\right] .
$$

If the component equations of this system are added, then a scalar conservation law for $w:=u+v$ with cubic flux is obtained:

$$
\partial_{t} w+\partial_{x} w^{3}=0
$$

The conservation law satisfied by the sum $w=u+v$ suggests the following entropy pair:

$$
\eta(U)=\frac{w^{2}}{2}, \quad q(U)=\frac{3}{4} w^{3} .
$$

The eigenvalues of $A(U)$ are $\lambda_{1}(U)=w^{2}$ and $\lambda_{2}(U)=3 w^{2}$, while corresponding eigenvectors are

$$
r_{1}(U)=\left[\begin{array}{c}
1 \\
-1
\end{array}\right], \quad r_{2}(U)=\left[\begin{array}{l}
1 \\
1
\end{array}\right] .
$$

Therefore, the system is always hyperbolic and it is strictly hyperbolic if $u+v \neq 0$. The characteristic field $r_{1}(U)$ is linearly degenerate and the corresponding simple waves are contact discontinuities that link states $U^{ \pm}$such that

$$
w^{+}=w^{-}
$$

The characteristic field $r_{2}(U)$ satisfies

$$
\nabla \lambda_{2}(U) \cdot r_{2}(U) \neq 0 \text { if } u+v \neq 0 .
$$

The corresponding rarefaction waves link two states $U^{ \pm}$satisfying

$$
u^{+}-v^{+}=u^{-}-v^{-}
$$

In order to set unambiguously the jump conditions, we consider the following regularized system:

$$
\begin{aligned}
& \partial_{t} u+2(u+v)^{2} u_{x}+(u+v)^{2} v_{x}=\epsilon_{1}(u+v)_{x x}+\delta_{1} \epsilon_{1}^{2}(u+v)_{x x x} \\
& \partial_{t} v+(u+v)^{2} u_{x}+2(u+v)^{2} v_{x}=\epsilon_{2}(u+v)_{x x}+\delta_{2} \epsilon_{1}^{2}(u+v)_{x x x}
\end{aligned}
$$

where $\delta_{i}, i=1,2$ are positive constants. If these two constants are such that:

$$
\gamma:=\delta_{1} s_{1}^{2}+\delta_{2} s_{2}^{2}=\frac{\delta_{1} s_{1}^{2}-\delta_{2} s_{2}^{2}}{s_{1}-s_{2}}
$$

where

$$
s_{i}=\frac{\epsilon_{i}}{\epsilon_{1}+\epsilon_{2}}, \quad i=1,2,
$$

then the traveling waves of the regularized system can be explicitly computed. A similar situation happens in the context of Navier-Stokes equations: when the Prandtl number is $3 / 4$ then a simple exact solution of the viscous profiles can be computed: see [41].

Notice first that the regularized equation satisfied by $w$ is:

$$
w_{t}+\left(w^{3}\right)_{x}=\epsilon w_{x x}+\gamma \epsilon^{2} w_{x x x}
$$


where $\epsilon=\epsilon_{1}+\epsilon_{2}$. Next, we look for a number $\lambda$ and a pair of functions $(u(y), v(y))$ with

$$
y=\frac{x-\lambda t}{\epsilon}
$$

solving (2.5) as well as the boundary conditions:

$$
\begin{array}{lc}
\lim _{y \rightarrow \pm \infty} u(y)=u^{ \pm}, & \lim _{y \rightarrow \pm \infty} v(y)=v^{ \pm}, \\
\lim _{y \rightarrow \pm \infty} u^{\prime}(y)=0, & \lim _{y \rightarrow \pm \infty} v^{\prime}(y)=0, \\
\lim _{y \rightarrow \pm \infty} u^{\prime \prime}(y)=0, & \lim _{y \rightarrow \pm \infty} v^{\prime \prime}(y)=0 .
\end{array}
$$

Some easy computations show that $u$ and $v$ have to satisfy:

$$
\begin{aligned}
& -\lambda u^{\prime}+2(u+v)^{2} u^{\prime}+(u+v)^{2} v^{\prime}=s_{1}(u+v)^{\prime \prime}+\delta_{1} s_{1}^{2}(u+v)^{\prime \prime \prime}, \\
& -\lambda v^{\prime}+(u+v)^{2} u^{\prime}+2(u+v)^{2} v^{\prime}=s_{2}(u+v)^{\prime \prime}+\delta_{2} s_{2}^{2}(u+v)^{\prime \prime} .
\end{aligned}
$$

The function $w=u+v$ satisfies the equation:

$$
-\lambda w^{\prime}+3 w^{2} w^{\prime}=w^{\prime \prime}+\gamma w^{\prime \prime \prime},
$$

and thus it is a traveling wave of (2.7), and these traveling waves can be exactly computed: see [29] and the references therein for the theory of nonclassical shocks.

On the other hand, thanks to (2.6), $z=u-v$ satisfies the equation

$$
-\lambda z^{\prime}+w^{2} z^{\prime}=\left(s_{1}-s_{2}\right)\left(w^{\prime \prime}+\gamma w^{\prime \prime \prime}\right)
$$

and, thus,

$$
\left(-\lambda+w^{2}\right) z^{\prime}=\left(s_{1}-s_{2}\right)\left(-\lambda w^{\prime}+3 w^{2} w^{\prime}\right)
$$

which allows us to compute $z$ (and thus $u$ and $v$ ) once $w$ is known. In particular, it can be shown that two states $U^{ \pm}$can be connected by a traveling wave if $w^{ \pm}$can be connected, in turn, via the equation (2.9). Consequently, $u^{ \pm}, v^{ \pm}$satisfy the relations

$$
\begin{aligned}
u^{+}= & \frac{1}{2}\left(w^{+}+u^{-}-v^{-}+\left(s_{1}-s_{2}\right)\left(3\left(w^{+}-w^{-}\right)\right.\right. \\
& \left.\left.+\sqrt{\lambda}\left(\log \left(\left|\frac{w^{+}-\sqrt{\lambda}}{w^{+}+\sqrt{\lambda}}\right|\right)-\log \left(\left|\frac{w^{-}-\sqrt{\lambda}}{w^{-}+\sqrt{\lambda}}\right|\right)\right)\right)\right), \\
v^{+}= & w^{+}-u^{+},
\end{aligned}
$$

where

$$
\lambda=\left(w^{+}\right)^{2}+w^{+} w^{-}+\left(w^{-}\right)^{2} .
$$

Once the traveling waves have been computed, the physically meaningful jump conditions are derived.

Figure 1 shows the Hugoniot curve of a given left state and the components of the right states $U r=\left(u_{r}, v_{r}\right)$ as a function of $w_{r}=u_{r}+v_{r}$. It can be noticed that these components tend to $\pm \infty$ as $w_{r} \rightarrow 0^{+}$. Therefore the Hugoniot curve of a state satisfying $w_{r}>0$ cannot pass through $w=0$. We will consider this system in the region $u+v>0$ in which the first characteristic field is genuinely nonlinear. 

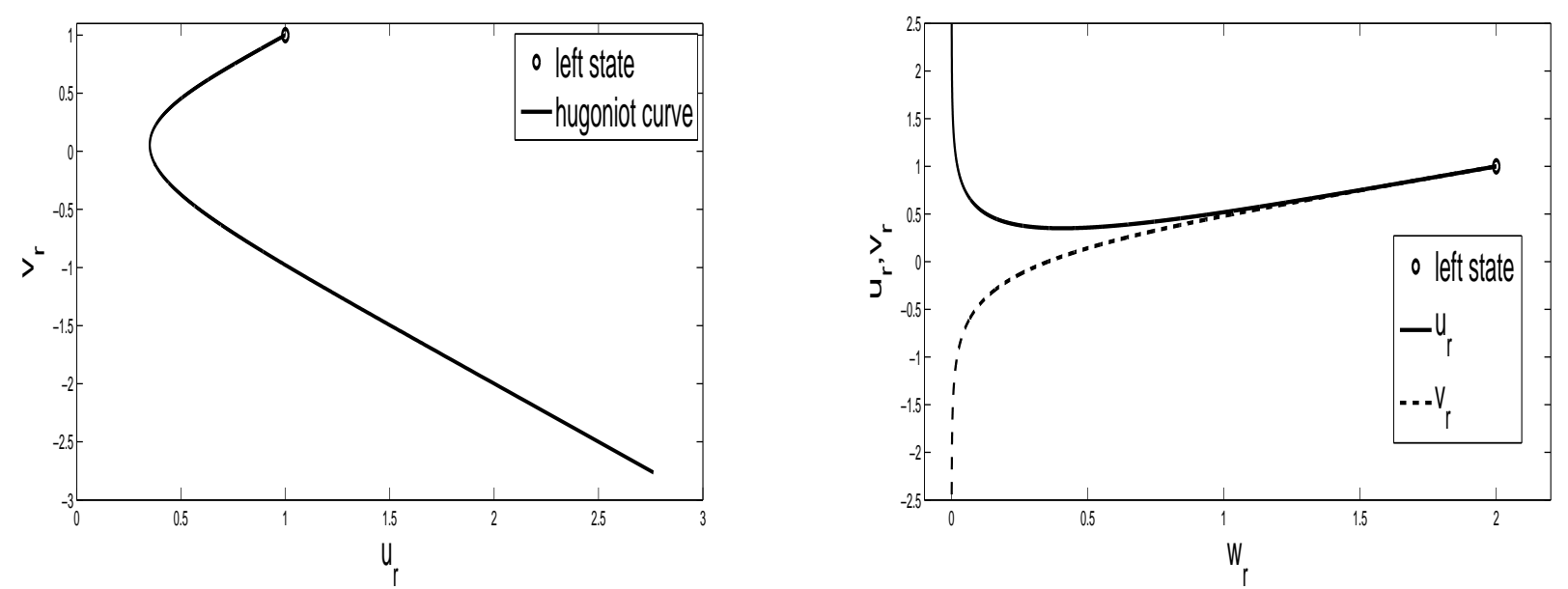

Fig. 1: Rankine-Hugoniot curve of the state $U_{l}=[1,1]$ (left), $u$ and $v$ components of the right states as a function of $w_{r}$ (right).

\subsection{The modified shallow water system}

\subsubsection{Formulation of the model}

In [8] the following system was considered

$$
\begin{aligned}
& h_{t}+q_{x}=0, \\
& q_{t}+\left(\frac{q^{2}}{h}\right)_{x}+q h h_{x}=0,
\end{aligned}
$$

where $q=u h$. This is a modification of the shallow water system in which the gravity has been replaced by the discharge in the pressure term of the momentum equation. Although this system does not come from a physical model, it is useful to test numerical methods for nonconservative systems, since it presents the difficulties related to the presence of nonconservative products arising in many physically relevant models but it is simple enough to be able to compute the solutions of the Riemann problems.

The system can be written in the form (1.1) with

$$
A(U)=\left[\begin{array}{cc}
0 & 1 \\
-u^{2}+u h^{2} & 2 u
\end{array}\right] .
$$

The eigenvalues of $A(U)$ are $\lambda_{1}(U)=u-h \sqrt{u}$ and $\lambda_{2}=u+h \sqrt{u}$, and

$$
R_{i}(U)=\left[\begin{array}{c}
1 \\
\lambda_{i}(U)
\end{array}\right], \quad i=1,2,
$$

are associated eigenvectors. The system is strictly hyperbolic if $u>0$ and $h \neq 0$. On the other hand, some straightforward calculations show that:

$$
\nabla \lambda_{1}(U) \cdot R_{1}(U)=-2 \sqrt{u}+\frac{h}{2}, \quad \nabla \lambda_{2}(U) \cdot R_{2}(U)=2 \sqrt{u}+\frac{h}{2} .
$$

Therefore the characteristic fields are genuinely nonlinear if $h \neq \pm 4 \sqrt{u}$. We consider the system in the region

$$
\Omega=\{(h, q) \mid 0<u, 0<h<4 \sqrt{u}\},
$$


in which the system is strictly hyperbolic and the characteristic fields are genuinely nonlinear. The Riemann invariants are given by $\sqrt{u}+h / 2=$ const and $\sqrt{u}-h / 2=$ const.

In [8] a specific family of paths was chosen to set the definition of weak solutions in the presence of shocks. Here, we proceed in a different way: we show that the system can be written in conservative form after a change of variables. We assume here that the weak of solutions of this conservative reformulation are the correct ones and thus the right jump condition is the corresponding Rankine-Hugoniot condition. Indeed, by using the identity

$$
q\left(\frac{h^{2}}{2}\right)_{x}=\left(q \frac{h^{2}}{2}\right)_{x}+\left(\frac{h^{3}}{6}\right)_{t},
$$

the system reads

$$
\begin{aligned}
& h_{t}+q_{x}=0, \\
& \left(q+\frac{h^{3}}{6}\right)_{t}+\left(\frac{q^{2}}{h}+q \frac{h^{2}}{2}\right)_{x}=0 .
\end{aligned}
$$

The corresponding Rankine-Hugoniot conditions are as follows:

$$
\begin{aligned}
& -\sigma\left(h_{+}-h_{-}\right)+q_{+}-q_{-}=0, \\
& -\sigma\left(q_{+}+\frac{h_{+}^{3}}{6}-q_{-}-\frac{h_{-}^{3}}{6}\right)+\frac{q_{+}^{2}}{h}{ }_{+}-\frac{q_{-}^{2}}{h}-+\frac{q_{+} h_{+}^{2}}{2}-\frac{q_{-} h_{-}^{2}}{2}=0,
\end{aligned}
$$

where $q_{ \pm}$and $h_{ \pm}$are the right and left limits, and $\sigma$ the shock speed.

Eliminating $\sigma$ and defining

$$
u_{ \pm}=\frac{q_{ \pm}}{h_{ \pm}}, \quad \tilde{u}=\frac{u_{+}-u_{-}}{h_{+}-h_{-}}
$$

the following equation is obtained

$$
6 h_{+} h_{-} \tilde{u}^{2}-h_{+}\left(2 h_{+}+h_{-}\right)\left(h_{+}-h_{-}\right) \tilde{u}-2 u_{-}\left(h_{+}^{2}+h_{-}^{2}+h_{+} h_{-}\right)=0,
$$

and thus

$$
\begin{aligned}
\tilde{u}= & \frac{1}{12 h_{+} h_{-}}\left(h_{+}\left(2 h_{+}+h_{-}\right)\left(h_{+}-h_{-}\right)\right. \\
& \left. \pm \sqrt{h_{+}^{2}\left(2 h_{+}+h_{-}\right)^{2}\left(h_{+}-h_{-}\right)^{2}+48 u_{-} h_{+} h_{-}\left(h_{+}^{2}+h_{-}^{2}+h_{+} h_{-}\right)}\right) .
\end{aligned}
$$

These two values of $\tilde{u}$ allow us to obtain, given $h_{+}, h_{-}$and $u_{-}$, the possible values of $u_{+}$, that is, the Rankine-Hugoniot curves of a given state $U^{-}$. It can be easily checked that 1-shocks correspond to the choice of the minus sign and 2-shocks to the plus sign. Moreover, if Lax entropy condition is assumed, the entropy 1 -shocks correspond to $0<h_{+} \leq h_{-}$and the entropy 2 -shocks to $0<h_{-} \leq h_{+}$. The Rankine-Hugoniot curves of different left states belonging to $\Omega$ are depicted in Figure 2 together with the boundary of $\Omega$. As the Figure seems to suggest, it can be checked that the Rankine-Hugoniot curves of a given state $U^{-} \in \Omega$ lie in $\Omega$.

\subsection{The two layer shallow water model}

We consider finally the one-dimensional two layer shallow water system that governs the evolution of two superposed shallow layers of immiscible fluids with constant densities $\rho_{i}, i=1,2\left(\rho_{1}<\rho_{2}\right)$ over a flat bottom:

$$
\begin{aligned}
& \frac{\partial h_{1}}{\partial t}+\frac{\partial q_{1}}{\partial x}=0, \\
& \frac{\partial q_{1}}{\partial t}+\left(\frac{q_{1}^{2}}{h_{1}}+\frac{g}{2} h_{1}^{2}\right)_{x}=-g h_{1} \frac{\partial h_{2}}{\partial x}, \\
& \frac{\partial h_{2}}{\partial t}+\frac{\partial q_{2}}{\partial x}=0, \\
& \frac{\partial q_{2}}{\partial t}+\left(\frac{q_{2}^{2}}{h_{2}}+\frac{g}{2} h_{2}^{2}\right)_{x}=-\frac{\rho_{1}}{\rho_{2}} g h_{2} \frac{\partial h_{1}}{\partial x},
\end{aligned}
$$




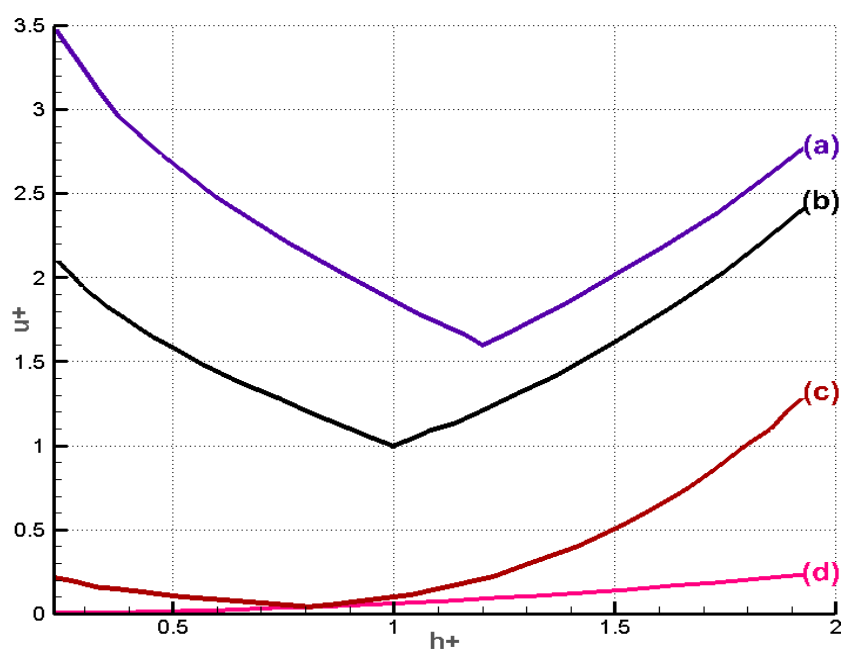

Fig. 2: Rankine-Hugoniot curves: curve (a) for $\left(h_{-}, u_{-}\right)=(1.2,1.6)$, curve (b) for $\left(h_{-}, u_{-}\right)=$ $(1 ., 1$.$) , curve (c) for \left(h_{-}, u_{-}\right)=(0.8,0.04)$. The curve (d) is defined by $h=4 \sqrt{u}$ which is the boundary of the domain defined by Equation (2.14)

where $h_{i}$ and $q_{i}$ denote respectively the thickness and the mass-flow of the $i$-th layer, and $g$ is the gravity.

This system can be written in the form (1.1) with

$$
U=\left[\begin{array}{l}
h_{1} \\
q_{1} \\
h_{2} \\
q_{2}
\end{array}\right], \quad A(U)=\left[\begin{array}{cccc}
0 & 1 & 0 & 0 \\
-u_{1}^{2}+c_{1}^{2} & 2 u_{1} & c_{1}^{2} & 0 \\
0 & 0 & 0 & 1 \\
r c_{2}^{2} & 0 & -u_{2}^{2}+c_{2}^{2} & 2 u_{2}
\end{array}\right]
$$

where $r=\rho_{1} / \rho_{2}, u_{i}=q_{i} / h_{i}$, and $c_{i}=\sqrt{g h_{i}}, i=1,2$.

In order to set the jump condition, we consider the following system with vanishing diffusion:

$$
\begin{aligned}
& \frac{\partial h_{1}}{\partial t}+\frac{\partial q_{1}}{\partial x}=\epsilon_{1}\left(h_{1}\right)_{x x} \\
& \frac{\partial q_{1}}{\partial t}+\left(\frac{q_{1}^{2}}{h_{1}}+\frac{g}{2} h_{1}^{2}\right)_{x}+g h_{1} \frac{\partial h_{2}}{\partial x}=\epsilon_{2}\left(q_{1}\right)_{x x} \\
& \frac{\partial h_{2}}{\partial t}+\frac{\partial q_{2}}{\partial x}=\epsilon_{3}\left(h_{2}\right)_{x x} \\
& \frac{\partial q_{2}}{\partial t}+\left(\frac{q_{2}^{2}}{h_{2}}+\frac{g}{2} h_{2}^{2}\right)_{x}+\frac{\rho_{1}}{\rho_{2}} g h_{2} \frac{\partial h_{1}}{\partial x}=\epsilon_{4}\left(q_{2}\right)_{x x} .
\end{aligned}
$$

The corresponding weak solutions depend on the way that the four parameters $\epsilon_{i}, i=1, \ldots, 4$, tend to 0 . The viscous terms coming from the physics of the problem only appear in the momentum equations, so that $\epsilon_{1}=0$ and $\epsilon_{3}=0$. On the other hand, the viscosity coefficient of the water of both water layers is similar, so that $\epsilon_{2} \approx \epsilon_{4}$ (see [9]). Nevertheless, for numerical purposes in Subsection 4.4 we consider the case:

$$
\epsilon_{2}=\epsilon_{4}=\epsilon, \quad \epsilon_{1}=\epsilon_{3}=\mu \epsilon,
$$

with $\epsilon>0$ and $\mu \geq 0$. Although the choice $\mu=0$ is the only one which is consistent with the physics of the problem, the numerical method introduced in next section is unstable for this choice due to the lack of viscosity in the first and third equations. Therefore, decreasing values of $\mu$ will be considered in order to approach the physically relevant solutions. 


\section{Schemes with well-controlled dissipation}

\subsection{Semi-discrete formulation}

In this section we adapt the class of finite difference schemes introduced in [17] for systems of conservation laws to numerically approximate discontinuous solutions of nonconservative equations with added vanishing diffusion and (possibly) dispersion, as stated in (1.2). Without loss of generality and for the sake of simplicity, we consider a uniform mesh $\left\{x_{i}\right\}_{i \in \mathbb{Z}}$ with mesh size $\Delta x=x_{i+1}-x_{i}$. The time-step and the time level at step $n$ are denoted by $\Delta t$ and $t_{n}=n \Delta t$, respectively. In order to introduce the main ideas, we consider a nonconservative system with linear vanishing diffusion and dispersion terms, i.e.

$$
U_{t}+A(U) U_{x}=\epsilon B U_{x x}+\delta \epsilon^{2} C U_{x x x}
$$

For such a system, we introduce the semi-discrete finite difference scheme

$$
\begin{aligned}
& \frac{d U_{i}}{d t}+\frac{1}{\Delta x} A\left(U_{i}\right)\left(\sum_{j=-p}^{p} \alpha_{j} U_{i+j}\right) \\
& =\frac{c}{\Delta x} B\left(\sum_{j=-p}^{p} \beta_{j} U_{i+j}\right)+\frac{\delta c^{2}}{\Delta x} C\left(\sum_{j=-p}^{p} \gamma_{j} U_{i+j}\right),
\end{aligned}
$$

in which $U_{i}(t)=U\left(x_{i}, t\right)$ represents the nodal value and $c=c(t) \geq 0$ is a time-dependent parameter to be determined. By applying a Taylor series expansion to $u\left(x_{i}+j \Delta x, t\right)$ up to terms of the order $O\left(\Delta x^{2 p+1}\right)$, where $u$ is a smooth solution of the system, the following consistency conditions are found to determine the coefficients $\alpha_{i}, \beta_{i}, \gamma_{i}$ :

$$
\begin{aligned}
& \sum_{j=-p}^{j=p} j \alpha_{j}=1, \quad \sum_{j=-p}^{j=p} j^{s} \alpha_{j}=0, \quad s \neq 1 \text { and } 0 \leq s \leq 2 p, \\
& \sum_{j=-p}^{j=p} j^{2} \beta_{j}=2, \quad \sum_{j=-p}^{j=p} j^{s} \beta_{j}=0, \quad s \neq 2 \text { and } 0 \leq s \leq 2 p, \\
& \sum_{j=-p}^{j=p} j^{3} \gamma_{j}=6, \quad \sum_{j=-p}^{j=p} j^{s} \gamma_{j}=0, \quad s \neq 3 \text { and } 0 \leq s \leq 2 p .
\end{aligned}
$$

Observe that these conditions define a set of three linear systems of $(2 p+1)$ equations and $(2 p+1)$ unknowns with Vandermonde matrices that can be explicitly solved to obtain the three sets of coefficients.

However, it is well-known that Vandermonde matrices are ill-conditioned and can lead to large errors in the coefficients. In order to avoid this, let us compute the solutions of these systems. Notice first that, if $\alpha_{-p}, \ldots, \alpha_{p}$ satisfy (3.3) then the $2 p+1$ point numerical differentiation formula:

$$
f^{\prime}(0) \cong D_{2 p+1}^{1}(f)=\sum_{j=-p}^{p} \alpha_{j} f(j)
$$

is exact for polynomials of degree lower or equal to $2 p$. Therefore, the linear system has a unique solution which is given by the weights of the interpolation-differentiation formula (3.6) with points $-p, \ldots, 0, \ldots, p$, that is, $\alpha_{j}=l_{j}^{\prime}(0)$ for $j=-p, \ldots, p$, where

$$
l_{j}(x)=\frac{(x+p) \ldots(x-j+1)(x-j-1) \ldots(x-p)}{(j+p) \ldots(1)(-1) \ldots(j-p)}, \quad j=-p, \ldots, p,
$$


are the Lagrange basis polynomials. Tedious but straightforward calculations allow us to obtain the explicit expression of these coefficients:

$$
\begin{aligned}
\alpha_{j} & =(-1)^{j+1} \frac{(p !)^{2}}{j(p+j) !(p-j) !}, \quad j=1, \ldots, p, \\
\alpha_{0} & =0, \\
\alpha_{j} & =-\alpha_{-j}, \quad j=-1, \ldots,-p .
\end{aligned}
$$

Analogously, the solutions of (3.4) and (3.5) can be interpreted as the weights of the interpolationdifferentiation formulas

$$
\begin{aligned}
& f^{\prime \prime}(0) \cong D_{2 p+1}^{2}(f)=\sum_{j=-p}^{p} \beta_{j} f(j), \\
& f^{\prime \prime \prime}(0) \cong D_{2 p+1}^{3}(f)=\sum_{j=-p}^{p} \gamma_{j} f(j),
\end{aligned}
$$

given by $\beta_{j}=l_{j}^{\prime \prime}(0), \gamma_{j}=l_{j}^{\prime \prime \prime}(0)$ for $j=-p, \ldots, p$. The expressions of these coefficients are as follows:

$$
\begin{aligned}
& \beta_{j}=(-1)^{j+1} \frac{2(p !)^{2}}{j^{2}(p+j) !(p-j) !}, \quad j=1, \ldots, p, \\
& \beta_{j}=\beta_{-j}, \quad j=-1, \ldots,-p, \\
& \beta_{0}=-2 \sum_{j=1} \beta_{j},
\end{aligned}
$$

and

$$
\begin{aligned}
& \gamma_{j}=(-1)^{j} \frac{6(p !)^{2}}{j(p+j) !(p-j) !} \sum_{1 \leq k \leq p, k \neq j} \frac{1}{k^{2}}, \quad j=1, \ldots, p, \\
& \gamma_{0}=0, \\
& \gamma_{j}=-\gamma_{-j}, \quad j=-1, \ldots,-p .
\end{aligned}
$$

For computational purposes, the formulas of the coefficients may be rewritten without factorial numbers by grouping adequately the factors.

For any integer $k$ let us define the following parameters that will be used in next section:

$$
A_{k}^{p}=\sum_{j=-p}^{j=p} \alpha_{j} j^{k}, \quad B_{k}^{p}=\sum_{j=-p}^{j=p} \beta_{j} j^{k}, \quad C_{k}^{p}=\sum_{j=-p}^{j=p} \gamma_{j} j^{k} .
$$

Observe that, for $0 \leq k \leq 2 p$, the values of these parameters vanish except $A_{1}^{p}=1, B_{2}^{p}=2$, and $C_{3}^{p}=6$. For $k$ greater than $2 p$ these parameters must be calculated numerically. These parameters can also be written in terms of the numerical differentiation formulas (3.6), (3.8), (3.9) as follows:

$$
A_{k}^{p}=D_{2 p+1}^{1}\left(x^{k}\right), \quad B_{k}^{p}=D_{2 p+1}^{2}\left(x^{k}\right), \quad C_{k}^{p}=D_{2 p+1}^{3}\left(x^{k}\right) .
$$

\subsection{Analysis via the equivalent equations}

Following [21,33] and, more recently, [17], we use the notion of equivalent equation in order to design our class of WCD schemes. A formal Taylor expansion in (3.2) allows us to derive the following equivalent equation:

$$
\begin{aligned}
U_{t}+A(U) U_{x}= & c \Delta x B U_{x x}+\delta c^{2} \Delta x^{2} C U_{x x x}-A(U)\left(\sum_{k=2 p+1}^{\infty} \frac{\Delta x^{k-1}}{k !} A_{k}^{p} U_{x}^{(k)}\right) \\
& +c B\left(\sum_{k=2 p+1}^{\infty} \frac{\Delta x^{k-1}}{k !} B_{k}^{p} U_{x}^{(k)}\right)+\delta c^{2} C\left(\sum_{k=2 p+1}^{\infty} \frac{\Delta x^{k-1}}{k !} C_{k}^{p} U_{x}^{(k)}\right),
\end{aligned}
$$


thus establishing that (3.2) is a first-order consistent method for (1.1) and a $2 p$ th-order consistent method for (3.1) with the choice $c \Delta x=\epsilon$.

As in $[17,32]$, the coefficient $c$ is chosen such that, at a discontinuity, the high-order terms of the discretization error are well-controlled, i.e. bounded by the underlying low-order terms. To this end, let us consider a single discontinuity

$$
U(x, t)= \begin{cases}U^{-} & \text {if } x \leq \sigma t \\ U^{+} & \text {otherwise }\end{cases}
$$

satisfying Dal Maso-LeFloch-Murat's jump conditions [15]:

$$
\int_{0}^{1} A\left(\Phi\left(s, U^{-}, U^{+}\right)\right) \partial_{s} \Phi\left(s, U^{-}, U^{+}\right) d s=\sigma[U],
$$

where $\sigma$ is the speed of propagation, $U^{ \pm}$are the left- and right-hand limit values, with

$$
[U]=U^{+}-U^{-} \text {. }
$$

Moreover, according to the theory of nonconservative hyperbolic systems introduced in [26], the map

$$
s \in[0,1] \mapsto \Phi\left(s, U^{-}, U^{+}\right)
$$

is, after a reparametrization, the profile of the traveling wave connecting $U^{-}$to $U^{+}$.

At the discontinuity the weak solution formally satisfies

$$
U_{x}^{(k)}=O\left(\frac{[U]}{\Delta x^{k}}\right)
$$

in the following sense: for a smooth function $v$ one has the equality

$$
v^{(k)}(x)=\lim _{\Delta x \rightarrow 0} \frac{\sum_{j=0}^{k}(-1)^{j}\left(\begin{array}{c}
k \\
j
\end{array}\right) v(x+(p-j) \Delta x)}{\Delta x^{k}},
$$

where $p=k / 2$ if $k$ is even and $p=(k+1) / 2$ if $p$ is odd. If now we compute the incremental ratio above for $U$ at $x=\sigma t$, we obtain:

$$
\frac{\sum_{j=0}^{k}(-1)^{j}\left(\begin{array}{c}
k \\
j
\end{array}\right) U(\sigma t+(p-j) \Delta x, t)}{\Delta x^{k}}=C_{k} \frac{[U]}{\Delta x^{k}},
$$

for some constant $C_{k}$. Analogously,

$$
A(U) U_{x}(\sigma t, t)=O\left(\frac{\sigma[U]}{\Delta x}\right) .
$$

In effect, for a smooth vectorial function $V$ one has:

$$
A(V(x)) V^{\prime}(x)=\lim _{\Delta x \rightarrow 0} \frac{1}{\Delta x} \int_{x-\frac{\Delta x}{2}}^{x+\frac{\Delta x}{2}} A(V(s)) V^{\prime}(s) d s .
$$

Notice that, if we replace $V$ by $U(\cdot, t)$ and $x$ by $\sigma t$ in the integral above, the integrand vanishes at every $s \neq \sigma t$. Nevertheless, within the Dal-Maso-LeFloch-Murat theory, the nonconservative product $A(U) U_{x}$ is understood in this case as a Dirac measure placed at $x=\sigma t$, whose mass is given by the integral:

$$
\int_{0}^{1} A\left(\Phi\left(s, U^{-}, U^{+}\right)\right) \partial_{s} \Phi\left(s, U^{-}, U^{+}\right) d s .
$$


In this sense:

$$
\frac{1}{\Delta x} \int_{\sigma t-\frac{\Delta x}{2}}^{\sigma t+\frac{\Delta x}{2}} A(U(s, t)) U_{x}(s, t) d s=\frac{1}{\Delta x} \int_{0}^{1} A\left(\Phi\left(s, U^{-}, U^{+}\right)\right) \partial_{s} \Phi\left(s, U^{-}, U^{+}\right) d s=\frac{\sigma[U]}{\Delta x},
$$

where (3.16) has been used.

We now use formally (3.17) and (3.18) in (3.14), to compare the order of magnitude of the different terms:

$$
\begin{aligned}
\frac{d U}{d t}+\sigma \frac{[U]}{\Delta x}= & c B \frac{[U]}{\Delta x}+\delta c^{2} C \frac{[U]}{\Delta x} \\
& -S_{p}^{A} \sigma \frac{[U]}{\Delta x}+c S_{p}^{B} B \frac{[U]}{\Delta x}+\delta c^{2} S_{p}^{C} C \frac{[U]}{\Delta x}
\end{aligned}
$$

where

$$
S_{p}^{A}=\sum_{k=2 p+1}^{\infty} \frac{A_{k}^{p}}{k !}, \quad S_{p}^{B}=\sum_{k=2 p+1}^{\infty} \frac{B_{k}^{p}}{k !}, \quad S_{p}^{C}=\sum_{k=2 p+1}^{\infty} \frac{C_{k}^{p}}{k !} .
$$

These coefficients have to be also numerically computed, by using the numerical differentiation formulas (3.6), (3.8), and (3.9) as follows:

$$
S_{p}^{A}=\left.\left(D_{2 p+1}^{1}\left(e^{x}\right)-1\right)\right|_{x=A}, \quad S_{p}^{B}=\left.\left(D_{2 p+1}^{2}\left(e^{x}\right)-1\right)\right|_{x=B}, \quad S_{p}^{C}=\left.\left(D_{2 p+1}^{3}\left(e^{x}\right)-1\right)\right|_{x=C} .
$$

Equation (3.19) represents the balance of terms in the equivalent equation at a discontinuity. The parameter $c$ is thus chosen so that the high-order terms

$$
\text { h.o.t. }=S_{p}^{A} \sigma \frac{[U]}{\Delta x}-c S_{p}^{B} B \frac{[U]}{\Delta x}-\delta c^{2} S_{p}^{C} C \frac{[U]}{\Delta x},
$$

are dominated in amplitude by the leading low order terms

$$
\text { l.o.t. }=\sigma \frac{[U]}{\Delta x}-c B \frac{[U]}{\Delta x}-\delta c^{2} C \frac{[U]}{\Delta x},
$$

thus ensuring that the high-order terms of the equivalent equation are a small perturbation of the correct underlying diffusion and dispersion terms.

In practice, the speed $\sigma$ is not known a priori and, therefore, in order to estimate the high-order terms, we consider a Roe-type matrix, i.e. an intermediate matrix $A\left(U^{-}, U^{+}\right)$satisfying

$$
A\left(U^{-}, U^{+}\right)[U]=\int_{0}^{1} A\left(\Phi\left(s, U^{-}, U^{+}\right)\right) \partial_{s} \Phi\left(s, U^{-}, U^{+}\right) d s
$$

and we approximate the high-order and leading terms by

$$
\begin{gathered}
\text { h.o.t. }=S_{p}^{A} A\left(U^{-}, U^{+}\right) \frac{[U]}{\Delta x}-c S_{p}^{B} B \frac{[U]}{\Delta x}-\delta c^{2} S_{p}^{C} C \frac{[U]}{\Delta x}, \\
\text { l.o.t. }=A\left(U^{-}, U^{+}\right) \frac{[U]}{\Delta x}-c B \frac{[U]}{\Delta x}-\delta c^{2} C \frac{[U]}{\Delta x} .
\end{gathered}
$$

Note that, if $U^{ \pm}$can be linked by an admissible shock, then (3.24) and (3.16) imply:

$$
A\left(U^{-}, U^{+}\right)[U]=\sigma[U]
$$

In order to achieve this correct balance, a tolerance parameter $\tau<<1$ is fixed and $p$ is chosen so that

$$
\frac{\mid \text { h.o.t. } \mid}{\mid \text { l.o.t. } \mid}<\tau \text {. }
$$

We first derive an upper bound for the high-order terms. For every component $j=1, \ldots, N$, we write

$$
\mid \text { h.o.t. }\left.\right|_{j} \leq \frac{1}{\Delta x}\left(\left|S_{p}^{A}\left\|A\left(U^{-}, U^{+}\right)_{j}[U]|+c| S_{p}^{B}\right\| B_{j}[U]\right|+\delta c^{2}\left|S_{p}^{C} \| C_{j}[U]\right|\right) .
$$


Next, a lower bound for the low-order terms is obtained. To do so, we assume that $\delta>0$ and

$$
\left(B_{j}[U]\right) \cdot\left(C_{j}[U]\right) \geq 0, \quad \forall j, U^{ \pm} .
$$

These assumptions, that are satisfied in all the cases considered in this article, allow us to derive the inequalities:

$$
c\left|B_{j} \frac{[U]}{\Delta x}\right|+\delta c^{2}\left|C_{j} \frac{[U]}{\Delta x}\right| \leq \mid \text { l.o.t. }\left.\right|_{j}+\left|A\left(U^{-}, U^{+}\right)_{j} \frac{[U]}{\Delta x}\right|,
$$

and thus

$$
\mid \text { l.o.t. }\left.\right|_{j} \geq \frac{1}{\Delta x}\left(c\left|B_{j}[U]\right|+\delta c^{2}\left|C_{j}[U]\right|-\left|A\left(U^{-}, U^{+}\right)_{j}[U]\right|\right)
$$

Taking into account (3.28) and (3.31), we conclude that (3.27) is satisfied if we choose

$$
\begin{aligned}
c(1 & \left.-\frac{1}{\tau}\left|S_{p}^{B}\right|\right)\left|B_{j}[U]\right|+\delta c^{2}\left(1-\frac{1}{\tau}\left|S_{p}^{C}\right|\right)\left|C_{j}[U]\right| \\
& -\left(1+\frac{1}{\tau}\left|S_{p}^{A}\right|\right)\left|A\left(U^{-}, U^{+}\right)_{j}[U]\right| \geq 0 .
\end{aligned}
$$

Therefore, in order to design a scheme satisfying (3.27), it is enough to choose $c>0$ such that (3.32) is fulfilled.

We consider the two possible cases. If $C_{j}[U]>0$, (3.32) gives a quadratic relation to be satisfied by $c$. The second degree polynomial in the variable $c$ takes a negative value at $c=0$ and, if

$$
\left|S_{p}^{C}\right|<\tau
$$

it tends to $\infty$ as $c \rightarrow \infty$. In that case, it is enough to choose a value $c_{j}$ satisfying

$$
c_{j} \geq \xi
$$

where $\xi$ is the unique positive root of the polynomial.

If $C_{j}[U]=0$ but $B_{j}[U] \neq 0,(3.32)$ reduces to a linear relation and, if

$$
\left|S_{p}^{B}\right|<\tau
$$

it is enough to choose a value $c_{j}$ satisfying

$$
c_{j} \geq \frac{\left(1+\frac{1}{\tau}\left|S_{p}^{A}\right|\right)\left|A\left(U^{-}, U^{+}\right)_{j}[U]\right|}{\left(1-\frac{1}{\tau}\left|S_{p}^{B}\right|\right)\left|B_{j}[U]\right|} .
$$

Finally, once that $c_{1}, \ldots, c_{N}$ have been determined, we set the value of $c$ to

$$
c=\max _{1 \leq j \leq N} c_{j} .
$$

In practice, a value of the parameter, $c_{i+1 / 2}$, is computed whenever a discontinuity is detected at $x_{i+1 / 2}=\left(x_{i}+x_{i+1}\right) / 2$ by a smoothness indicator (based in the second order finite difference). Then, the value of the parameter $c$ is set to the maximum of these local values. On the other hand, the computation of a Roe matrix satisfying (3.24) for the family of paths given by the traveling waves may be very difficult and computationally expensive. In the numerical tests shown in Section 4, Roe matrices satisfying (3.24) for the family of straight segments

$$
\Phi\left(s, U^{-}, U^{+}\right)=U^{-}+s\left(U^{+}-U^{-}\right)
$$

are considered instead. The numerical results show that the values of $c$ computed with this approximation result in a balance of the high-order and leading-order terms. Nevertheless the value of $c$ may be large and thus the value of $\Delta x$ has to be small in order to avoid smeared shocks in the numerical solutions. 
We observe that it is always possible to take $p$ large enough so that (3.33) and (3.35) are satisfied. Indeed, the coefficients $S_{p}^{A}, S_{p}^{B}, S_{p}^{C}$ tend to 0 as $p \rightarrow \infty$, and this is checked as follows. Let us show the result for $S_{p}^{A}$ : the proof is similar for the other two coefficients. Taking into account (3.21), it is enough to prove:

$$
\lim _{p \rightarrow \infty} D_{2 p+1}^{1}\left(e^{x}\right)=1
$$

Given any function $f \in \mathcal{C}^{\infty}(\mathbb{R})$ and given an integer $p \geq 1$, there exits $\xi_{p} \in[-p, p]$ such that

$$
f^{\prime}(0)-D_{2 p+1}^{1}(f)=f^{\prime}(0)-q_{p}^{\prime}(0)=\frac{f^{(2 p+1)}\left(\xi_{p}\right)}{(2 p+1) !} \prod_{j=-p \ldots, p, j \neq 0}(-j),
$$

where $q_{p}$ represents the polynomial that interpolates the data:

$$
(-p, f(-p)), \ldots,(0, f(0)), \ldots(p, f(p)) .
$$

In the particular case of the exponential function, we obtain:

$$
\left|1-D_{2 p+1}^{1}\left(e^{x}\right)\right| \leq \frac{p !^{2} e^{p}}{(2 p+1) !}
$$

and the quotient criterion allows us to conclude the claimed result.

\subsection{Temporal integration}

The proposed semidiscrete schemes result in an ODE system of the form

$$
\frac{d \mathbf{U}}{d t}=\mathbf{L}(\mathbf{U}), \quad \mathbf{U}\left(t_{0}\right)=\mathbf{U}^{0},
$$

where $\mathbf{L}$ is an operator defined by the parameters used in semi-discrete form of the scheme. In our numerical tests we use one of the so-called total variation diminishing (TVD) Runge Kutta schemes for temporal integration introduced in [39]. This method is performed via three stages to solve equation (3.40), that is,

$$
\begin{aligned}
\mathbf{U}^{(1)} & =\mathbf{U}^{n}+\Delta t \mathbf{L}\left(\mathbf{U}^{n}\right), \\
\mathbf{U}^{(2)} & =\frac{3}{4} \mathbf{U}^{n}+\frac{1}{4} \mathbf{U}^{(1)}+\frac{1}{4} \Delta t \mathbf{L}\left(\mathbf{U}^{(1)}\right), \\
\mathbf{U}^{n+1} & =\frac{1}{3} \mathbf{U}^{n}+\frac{2}{3} \mathbf{U}^{(2)}+\frac{2}{3} \Delta t \mathbf{L}\left(\mathbf{U}^{(2)}\right) .
\end{aligned}
$$

\subsection{The condition on the time-step}

The goal of this paragraph is to derive a stability condition for practical purposes which takes into account both the hyperbolic part and the high-order terms of the system: a linear stability analysis is performed under some simplifying assumptions. A complete study of the fully discretized method is beyond the scope of the article and will be addressed in a next one. The stability condition found here imposes a restriction on the time step which is completely analogous to a standard CFL condition.

In order to estimate the requirement to be imposed to the time step for our method to be linearly stable, we apply von Neumann's analysis to the numerical scheme when $p$ is sufficiently large. We consider first the forward Euler method:

$$
U_{i}^{n+1}=U_{i}^{n}+\lambda\left(-A \sum_{j=-p}^{p} \alpha_{j} U_{i+j}^{n}+c B \sum_{j=-p}^{p} \beta_{j} U_{i+j}^{n}+\delta c^{2} C \sum_{j=-p}^{p} \gamma_{j} U_{i+j}^{n}\right),
$$


where $\lambda=\Delta t / \Delta x$, and $A, B, C$ are $N \times N$ constant matrices. Moreover, we suppose that these three matrices are diagonalizable and that they commute, i.e. they diagonalize in a common eigenvector basis. Let us denote by

$$
\mu_{k}^{A}, \quad \mu_{k}^{B}, \quad \mu_{k}^{C}, \quad k=1, \ldots, N,
$$

the eigenvalues of $A, B$, and $C$, ordered so that eigenvalues with the same index correspond to the same eigenvector of the chosen basis. The eigenvalues of $B$ and $C$ are supposed to be non-negative.

The Fourier modes satisfy:

$$
\hat{U}_{i}^{n+1}=G(\xi, \Delta x) \hat{U}_{i}^{n},
$$

where the symbol is given by:

$$
G(\xi, \Delta x)=I+\lambda\left(-A \sum_{j=-p}^{p} \alpha_{j} e^{-i j \Delta x \xi}+c B \sum_{j=-p}^{p} \beta_{j} e^{-i j \Delta x \xi}+\delta c^{2} C \sum_{j=-p}^{p} \gamma_{j} e^{-i j \Delta x \xi}\right) .
$$

Here $I$ is the identity matrix.

Using again the interpretation of the coefficients $\alpha_{j}, \beta_{j}, \gamma_{j}$ as the weights of interpolationdifferentiation formula, we have:

$$
\begin{aligned}
& \sum_{j=-p}^{p} \alpha_{j} e^{-i j \Delta x \xi}=D_{2 p+1}^{1}(g), \\
& \sum_{j=-p}^{p} \beta_{j} e^{-i j \Delta x \xi}=D_{2 p+1}^{2}(g), \\
& \sum_{j=-p}^{p} \gamma_{j} e^{-i j \Delta x \xi}=D_{2 p+1}^{3}(g),
\end{aligned}
$$

where $g(x)=e^{-i \Delta x \xi x}$. Therefore, if $p$ is large enough, we have:

$$
\begin{aligned}
& \sum_{j=-p}^{p} \alpha_{j} e^{-i j \Delta x \xi} \cong g^{\prime}(0)=-i \Delta x \xi, \\
& \sum_{j=-p}^{p} \beta_{j} e^{-i j \Delta x \xi} \cong g^{\prime \prime}(0)=-\Delta x^{2} \xi^{2}, \\
& \sum_{j=-p}^{p} \gamma_{j} e^{-i j \Delta x \xi} \cong g^{\prime \prime \prime}(0)=i \Delta x^{3} \xi^{3} .
\end{aligned}
$$

Therefore, the symbol can be estimated by:

$$
G(\xi, \Delta x) \cong I+\lambda\left(i \Delta x \xi A-c \Delta x^{2} \xi^{2} B+\delta c^{2} i \Delta x^{3} \xi^{3} C\right),
$$

and their eigenvalues by:

$$
\mu_{k}^{G}=1-\lambda c \Delta x^{2} \xi^{2} \mu_{k}^{B}+i \lambda\left(\Delta x \xi \mu_{k}^{A}+\delta c^{2} \Delta x^{3} \xi^{3} \mu_{k}^{C}\right), \quad k=1, \ldots, N .
$$

If $\mu_{k}^{B} \neq 0$, taking into account that the maximal wavenumber representable in the mesh is $\xi=\frac{\pi}{\Delta x}$, some straightforward calculations show that the inequality

$$
\frac{\Delta t}{\Delta x} \leq \frac{2 c \mu_{k}^{B}}{c^{2} \pi^{2}\left(\mu_{k}^{B}\right)^{2}+\left(\mu_{k}^{A}+\delta c^{2} \pi^{2} \mu_{k}^{C}\right)^{2}}, \quad k=1, \ldots, N,
$$

implies that

$$
\left|\mu_{k}^{G}\right| \leq 1, \quad 1 \leq k \leq N
$$


and the von Neumann stability condition is thus satisfied in the strict sense. Therefore:

$$
\left\|U^{n+1}\right\|_{2} \leq\left\|U^{n}\right\|_{2},
$$

where $\|\cdot\|_{2}$ is the $L^{2}$-norm.

Now, since the temporal integrator (3.41) can be represented as a convex combination of forward Euler methods, (3.43) is a stability condition also for the corresponding scheme. In effect, from the analysis above we derive:

$$
\begin{aligned}
\left\|U^{(1)}\right\|_{2} & \leq\left\|U^{n}\right\|_{2} \\
\left\|U^{(2)}\right\|_{2} & \leq \frac{3}{4}\left\|U^{n}\right\|_{2}+\frac{1}{4}\left\|U^{(1)}\right\| \leq\left\|U^{n}\right\|_{2}, \\
\left\|U^{n+1}\right\|_{2} & \leq \frac{1}{3}\left\|U^{n}\right\|_{2}+\frac{2}{3}\left\|U^{(2)}\right\| \leq\left\|U^{n}\right\|_{2} .
\end{aligned}
$$

If $\mu_{k}^{B}=0$ and we consider the time integrator (3.41), then some easy computations shows that:

$$
\hat{U}_{i}^{n+1}=\left(\frac{1}{3} I+\frac{1}{2} G(\xi, \Delta x)+\frac{1}{6} G(\xi, \Delta x)^{3}\right) \hat{U}_{i}^{n} .
$$

The eigenvalues of the symbol can be approached for large enough values of $p$ by:

$$
\mu_{k}^{G}=1-\frac{\left(\lambda \gamma_{k}\right)^{2}}{2}+i\left(\lambda \gamma_{k}-\frac{\left(\lambda \gamma_{k}\right)^{3}}{6}\right)
$$

where

$$
\gamma_{k}=\Delta x \xi \mu_{k}^{A}+\delta c^{2} \Delta x^{3} \xi^{3} \mu_{k}^{C}, \quad k=1, \ldots, N .
$$

Then, $\left|\mu_{k}^{G}\right| \leq 1$ is ensured if

$$
\lambda^{2} \gamma_{k}^{2} \leq 3
$$

The following stability condition is thus obtained:

$$
\frac{\Delta t}{\Delta x} \leq \frac{\sqrt{3}}{\pi\left|\mu_{k}^{A}+\delta c^{2} \pi^{2} \mu_{k}^{C}\right|}, \quad k=1, \ldots, N .
$$

Observe that the right-hand sides of (3.43) and (3.44) do not depend on $\Delta x$. Nevertheless, these conditions may be very restrictive in practice when $c$ is very large.

In the particular case of the Burgers coupled system, $A$ and $B$ the matrices do not commute. Nevertheless, the computation of the eigenvalues of the approximated symbol lead again to the stability condition (3.43) that, in this case, reads as follows:

$$
\frac{\Delta t}{\Delta x} \leq \frac{4 c}{4 c^{2} \pi^{2}+(u+v)^{2}}
$$

For the coupled cubic system, the matrices commute and the stability condition reads as follows:

$$
\frac{\Delta t}{\Delta x} \leq \frac{4 c}{4 c^{2} \pi^{2}+\left(3(u+v)^{2}+2 \delta c^{2} \pi^{2}\right)^{2}} .
$$

A deeper analysis may lead to sharper stability conditions if, for instance, the approximation for large values of $p$ is not applied and the eigenvalues of the symbol corresponding to the time integrator (3.41) are computed, or if nonlinear stability criteria are investigated. Nevertheless such an analysis is out of the scope of this paper. 


\section{Numerical experiments}

\subsection{The coupled Burgers equations}

In this section, we present numerical experiments that illustrate the approximation of different examples of nonconservative hyperbolic systems with the proposed WCD schemes. First of all, we consider the coupled Burgers system (2.1) with the notion of weak solution consistent with the viscous regularization $(2.2)$.

We consider first the Riemann problem, at $x=0$, with initial data

$$
U_{l}=\left[\begin{array}{c}
1 \\
0.25
\end{array}\right], \quad U_{r}=\left[\begin{array}{c}
2 \\
-1
\end{array}\right]
$$

for the coupled Burgers system (2.2). The exact solution of the Riemann problem consists of a stationary contact discontinuity and a shock. The numerical method (3.2) with $c=0.1, p=8$ is used to discretize the system in space and the 3rd order TVD RK is used for the time discretization. In all the numerical tests shown in this section, the boundary conditions are treated by adding $p$ ghost nodes on both sides of the computational interval whose values are set to the ones at the extreme nodes at every time step:

$$
U_{-j}^{n}=U_{0}^{n}, \quad U_{M+j}^{n}=U_{M}^{n}, \quad j=1, \ldots, p,
$$

where 0 and $M$ are the indexes of the first and last nodes.

Figure 3 shows the comparison of the numerical and the exact solutions for different mesh resolutions. Observe that the location of the shock and the intermediate state are correctly captured. This is further evidenced in a zoom of the solution around the shock, that is provided in Figure 4.

Figure 5 shows the errors corresponding to the numerical approximation of the intermediate state (between the contact and the shock) for different mesh steps and values of $p$ in logarithmic scale. In order to compare methods with similar shock-capturing properties, the horizontal axis corresponds to $\log (p \Delta x)$ (notice that the stencil of the method is $2 p \Delta x$ ). It can be observed that, for any considered value of $p$, the error first decreases with an order of accuracy of about 3 (which is expected, as the order of accuracy of the time stepping is 3) but then it stabilizes at a value (very small but non-zero), which decreases with higher values of $p$. Therefore, for a given value of $p$, the numerical method does not necessarily converge to the right weak solution as $\Delta x \rightarrow 0$ (notice the small but non-zero error): it converges to a slightly different weak solution that depends on the high-order terms of the equivalent equation. Nevertheless, if both $\Delta x$ tends to 0 and $p$ to $\infty$ the error tends to 0 , as it can be seen in Figure 6, where we show the error in logarithmic scale obtained with different values of $p$ using meshes such that $p \Delta x$ remain constant. Thus, we see that the WCD schemes will converge to the correct value if the formal order of approximation is arbitrarily high.

Note that the value of $c$, given by the WCD condition (3.36) is only a sufficient condition. It can lead to the overestimation of numerical viscosities as in the above example. For instance, with the values of $p=8$ and $\tau=0.1$ we obtain a value of $c=2.5$, which is clearly much higher than the necessary stabilizing viscosity. Nevertheless, the analysis in Section 3.2, indicates that a higher value of $c$ would increase robustness by improving the possibility of satisfying the WCD condition (3.36). We illustrate this fact by the following example.

We consider the Riemann problem for the coupled Burgers system with initial conditions

$$
U_{l}=\left[\begin{array}{c}
1 \\
0.25
\end{array}\right], \quad U_{r}=\left[\begin{array}{l}
-2 \\
-1
\end{array}\right] .
$$

In this case, the numerical method with $c=0.1$ gives very bad results even for very fine meshes: see Figure 7. Nevertheless, for $c=0.75$ a similar behavior as in the previous numerical example is observed: see Figure 8 that depicts the approximation obtained at $t=0.1$ with $p \Delta x=0.004$. The 

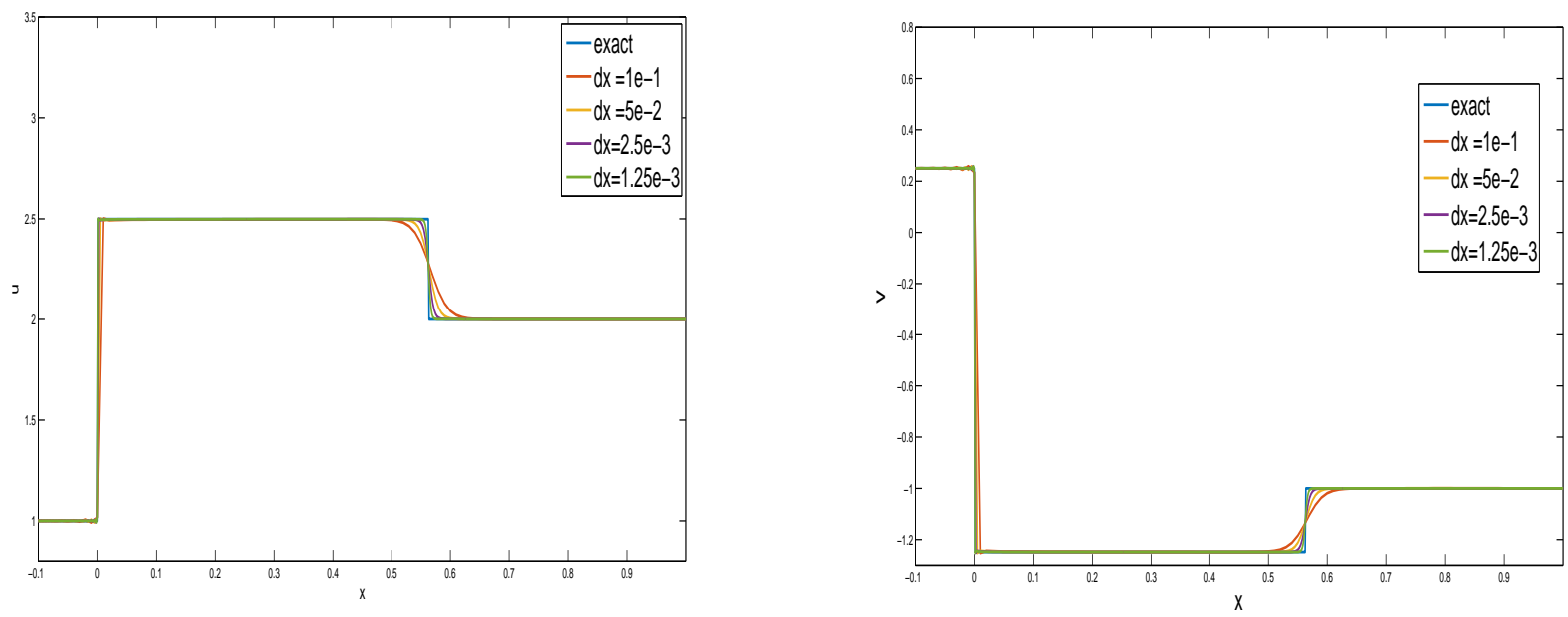

Fig. 3: Numerical results of the WCD method with $c=0.1, p=8$ for the coupled Burgers system with initial condition (4.1). Up: $u$, Down: $v$ ).
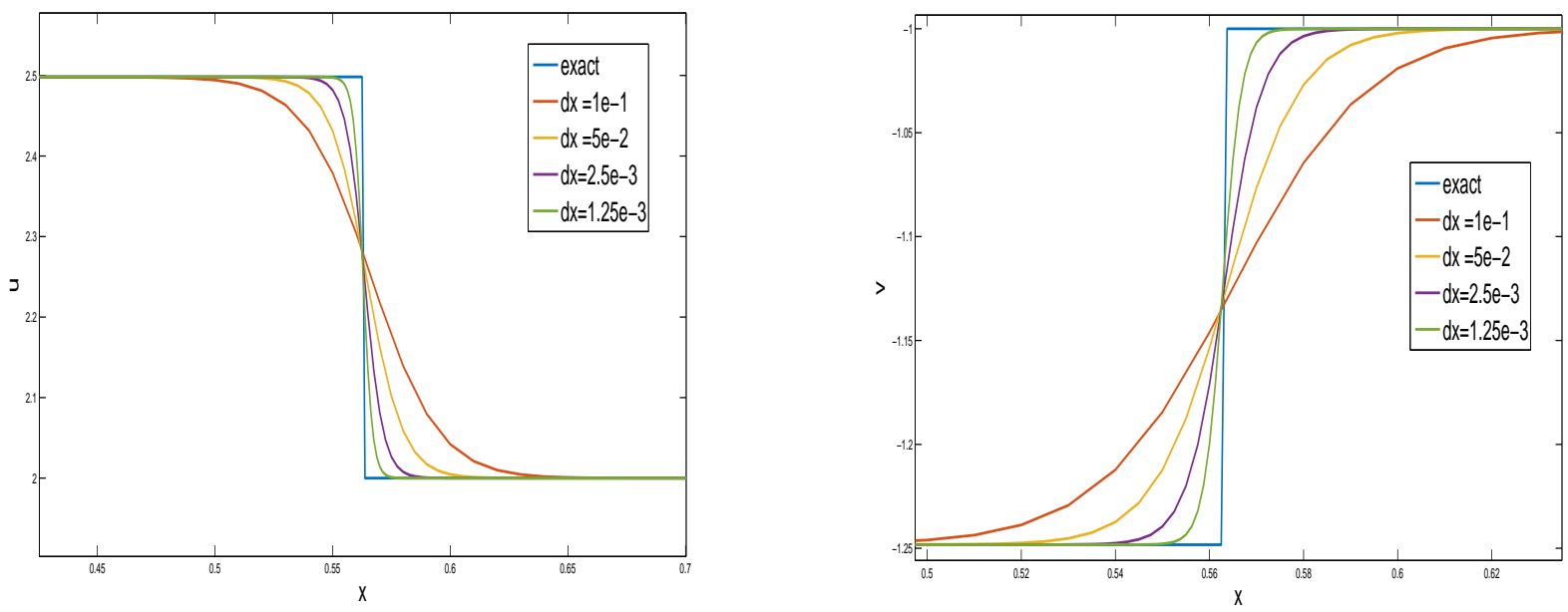

Fig. 4: Numerical results of the WCD method with $c=0.1, p=8$ for the coupled Burgers system with initial condition (4.1): zoom. Up: $u$, Down: $v$ ).

small over and undershots are due to the degeneracy of the viscous term: in a contact discontinuity $u+v$ remains constant and thus $\partial_{x x}^{2}(u+v)=0$.

In Figure 9 the exact Hugoniot curve of the left states that can be linked to the right state $U_{r}=(2,-1)$ by an admissible shock is compared with its numerical approximation obtained with $c=0.75, p=4$, and $\Delta x=0.004$ and illustrates the accurate approximation with this method.

\subsection{The coupled cubic equations}

Next, we consider the coupled cubic system (2.5) with parameters,

$$
s_{1}=2 / 3, \quad s_{2}=1 / 3, \quad \delta_{1}=1 / 2, \quad \delta_{2}=1 .
$$




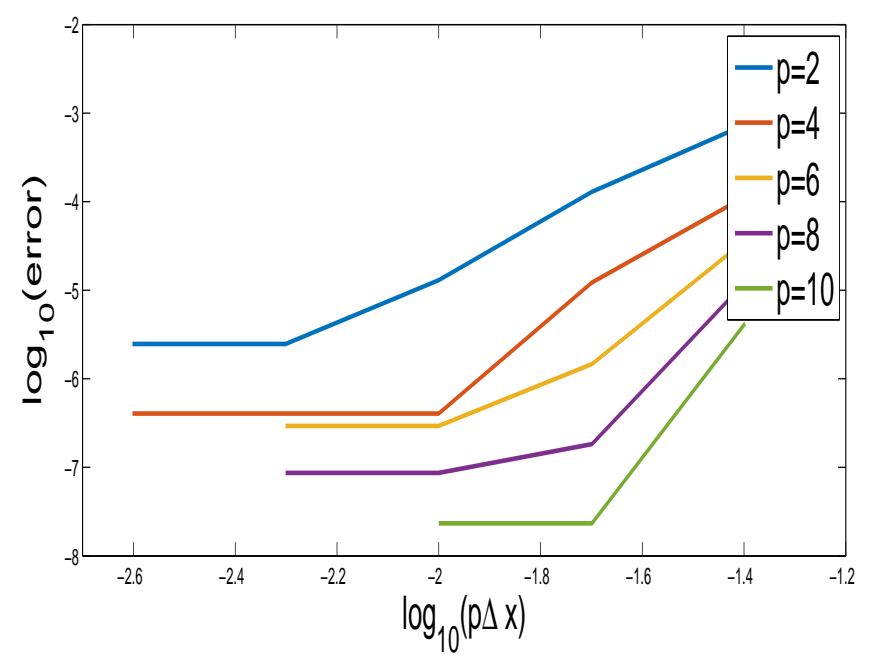

Fig. 5: Errors corresponding to the intermediate state of the Riemann problem corresponding to (4.1) in logarithmic scale. Horizontal axis: $\log _{10}(p \Delta x)$. Vertical axis: $\log _{10}($ error $)$.

We consider first the Riemann problem, at $x=0$, with initial data

$$
U_{l}=\left[\begin{array}{l}
1.5 \\
0.5
\end{array}\right], \quad U_{r}=\left[\begin{array}{l}
0.75 \\
0.25
\end{array}\right]
$$

The solution, corresponding to the underlying diffusive-dispersive regularization, consists of a contact discontinuity traveling at speed 4 and a shock whose speed is 7 . The numerical method (3.2) is used with $\Delta x=1 / 2500, \tau=10^{-1}, p=4$ to discretize the system in space, (3.34) is used to compute the parameter $c$, yielding a value of $c \cong 6$, and the 3rd order RK TVD method is considered for the time discretization. Figure 10 shows the comparison of the numerical and the exact solutions. Observe that the location of the shock and the intermediate state are correctly captured. A zoom of the region near the rear of the contact discontinuity is shown in Figure 11 in which the effects of the dispersion terms are apparent.

In the presence of a dispersion term, the choice of a parameter $c$ satisfying (3.34) is absolutely vital, in order to balance adequately the dispersion and the diffusion terms: Figure 12 shows the results obtained with the same parameters but using a value of $c$ not satisfying (3.34).

In Figure 13 the exact Hugoniot curve of the left states that can be linked to the right state $U_{r}=(.5,-.5)$ by an admissible shock is compared with its numerical approximation obtained with $\tau=0.1, p=4$, and $\Delta x=1 / 1500$ and shows that the WCD method is able to correctly approximate the small-scale dependent shock waves.

\subsection{The cubic flux equation}

In order to test the ability of the numerical method (3.2) to correctly capture non-classical shocks we consider the following Riemann problem for the cubic flux conservation law:

$$
\begin{aligned}
& u_{t}+\left(u^{3}\right)_{x}=0, \\
& u(x, 0)= \begin{cases}3, & x<0, \\
-3, & x>0 .\end{cases}
\end{aligned}
$$

The solution of this Riemann problem consistent with the regularization

$$
u_{t}+\left(u^{3}\right)_{x}=\epsilon u_{x x}+\frac{1}{2} \epsilon^{2} u_{x x x}
$$




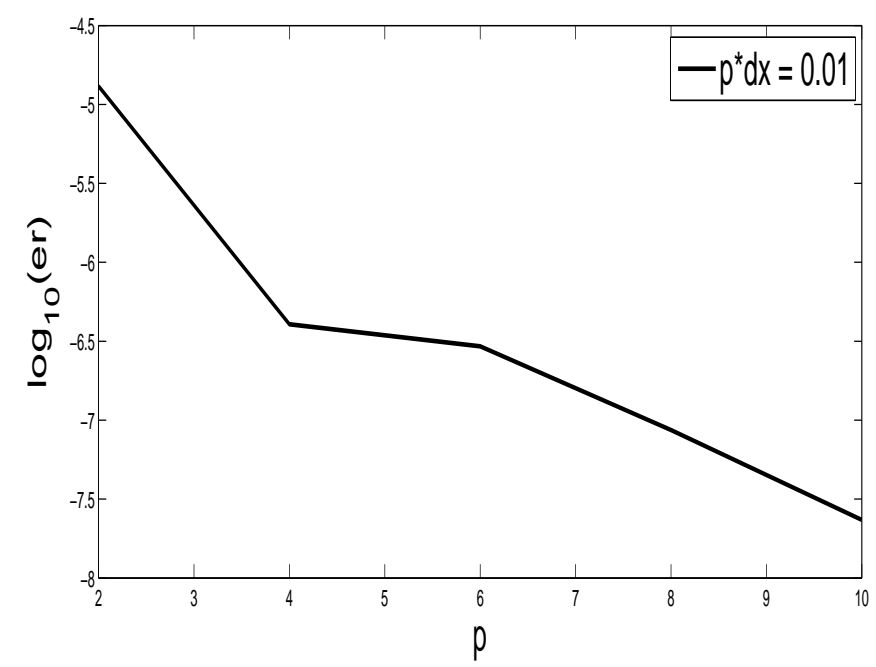

Fig. 6: Errors corresponding to the intermediate state of the Riemann problem corresponding to (4.1) in logarithmic scale with different values of $p$ and $p \Delta x=0.01$. Horizontal axis: $p$. Vertical axis: $\log _{10}($ error $)$.
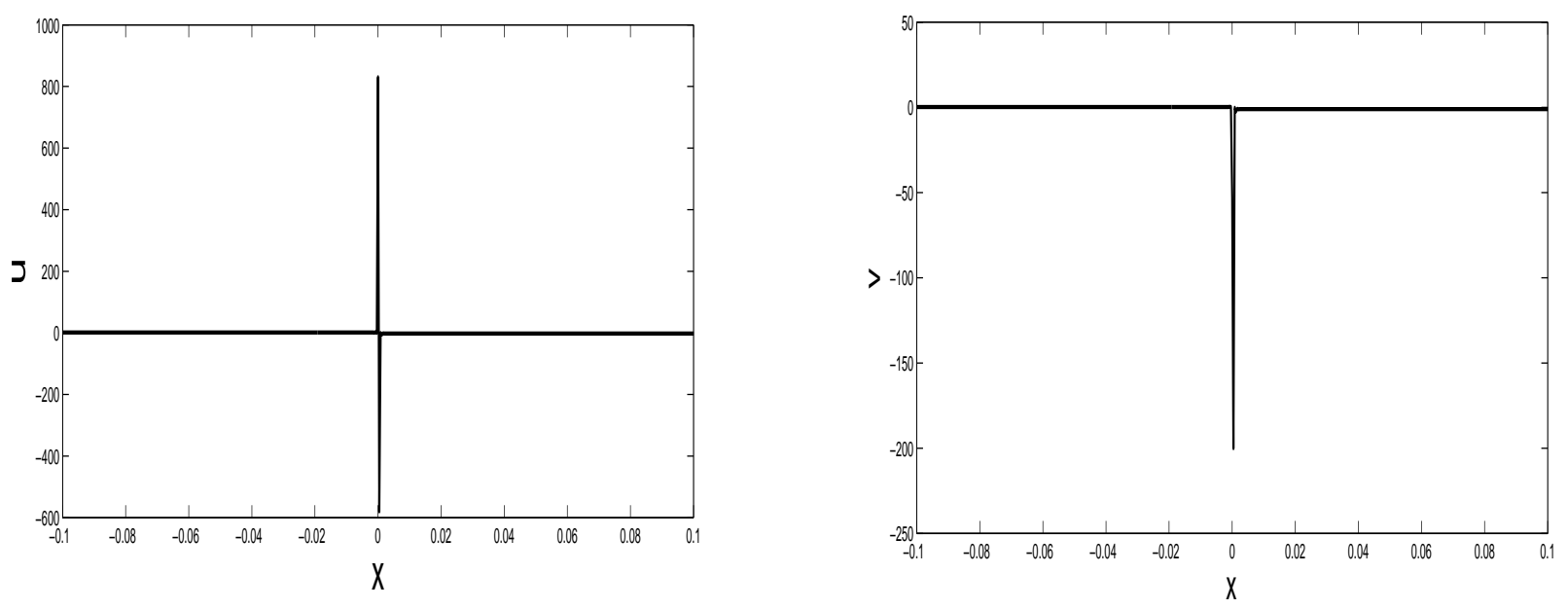

Fig. 7: Numerical results of the WCD method at $t=0.00027835$ for the coupled Burgers system with initial condition (4.2) with $c=0.1, p=2$ and $\Delta x=0.0004$ (Left: $u$, Right: $v$ ).

consists of a non-classical shock connecting $u_{l}=3$ to $u^{*}=-3+\frac{2}{3}$ and a rarefaction connecting $u^{*}$ to $u_{r}=-3$ : see [29].

To do this, we first write the equation in the form:

$$
u_{t}+3 u^{2} u_{x}=\epsilon u_{x x}+\frac{1}{2} \epsilon^{2} u_{x x x}
$$

that fits in the general framework (3.1), and then we apply the discretization (3.2) to obtain

$$
\frac{d u_{i}}{d t}+\frac{3}{\Delta x} u_{i}^{2}\left(\sum_{j=-p}^{p} \alpha_{j} u_{i+j}\right)=\frac{c}{\Delta x} \sum_{j=-p}^{p} \beta_{j} u_{i+j}+\frac{c^{2}}{2} \Delta x \sum_{j=-p}^{p} \gamma_{j} u_{i+j} .
$$



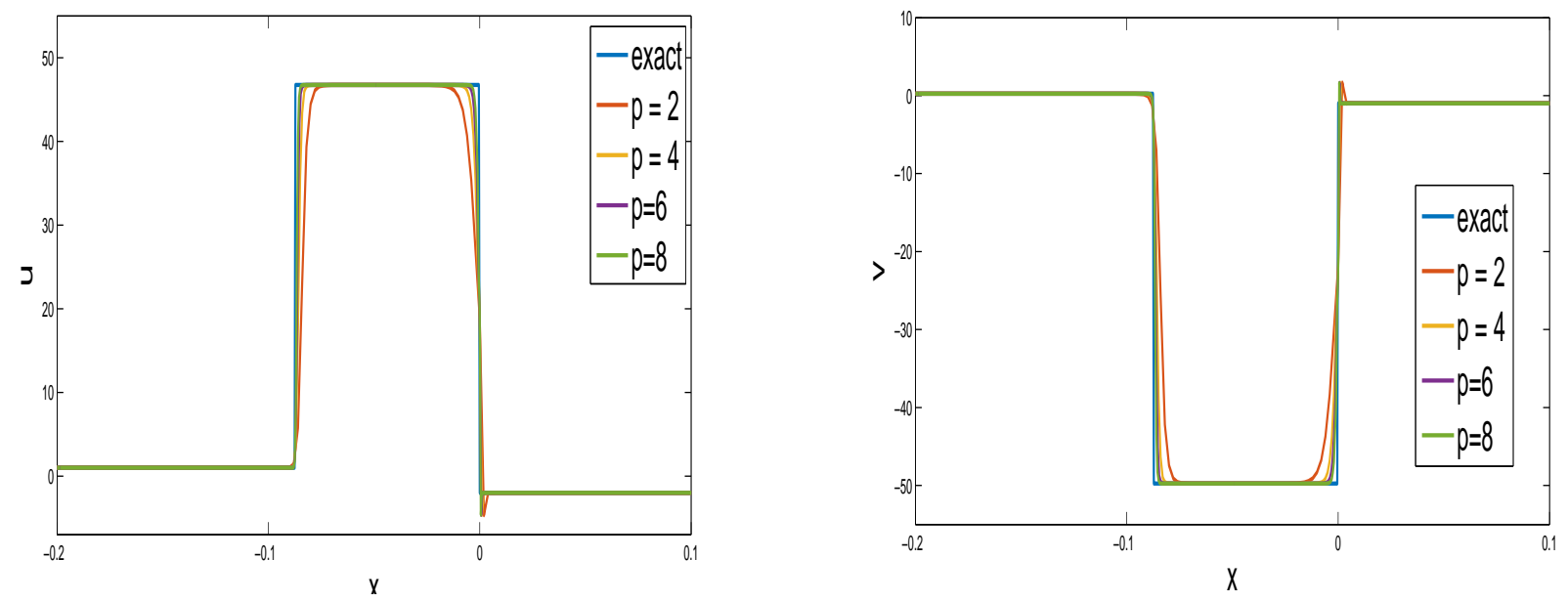

Fig. 8: Numerical results of the WCD method at $t=0.1$ for the coupled Burgers system with initial condition (4.2) with $c=0.75$, different values of $p$, and $p \Delta x=0.004$ (Left: $u$, Right: $v$ ).

The 3rd order TVD RK is considered for the time discretization.

Figure 14 shows the numerical results obtained at $t=0.03$ with $\Delta x=10^{-3}, \tau=10^{-1}$, and $p=6$. The numerical solutions are compared with the exact one: it can be seen that the non-classical shock is correctly captured. This numerical test is particularly challenging, as it deals with two major difficulties of the numerical approximation of nonlinear hyperbolic PDE: the convergence of numerical method in nonconservative form and the convergence to weak solutions involving non-classical shocks.

\subsection{The modified shallow water model}

In this section, the WCD scheme for nonconservative systems is applied to the following regularization of system (2.15) :

$$
\begin{aligned}
& h_{t}-\frac{h^{2}}{2} h_{x}+s_{x}=\epsilon_{1} h_{x x} \\
& s_{t}+\left(\frac{h s}{3}-\frac{5}{18} h^{4}-\frac{s^{2}}{h^{2}}\right) h_{x}+\left(\frac{2 s}{h}+\frac{h^{2}}{6}\right) s_{x}=\epsilon_{2} s_{x x}
\end{aligned}
$$

where $s=q+h^{3} / 6$.

The numerical results are compared to those obtained by using the WCD scheme developed by Ernest et al. in [17] for the same system in the conservative form. The third-order TVD Runge Kutta method is used for temporal integration.

We consider two different Riemann problems for the above system, involving shocks of different amplitude. The domain $[-1,1]$ and the following initial condition is considered

$$
h(0, x)=\left\{\begin{array}{l}
h_{L},-1 \leq x \leq 0 \\
h_{R}, \text { otherwise, }
\end{array}\right.
$$

and $u(0, x)=1$. We consider $\Delta x=1 / 500, \tau=0.01$, and $p=12$.

First, we consider (4.6) with $h_{L}=0.7$ and $h_{R}=0.5$ to realize shocks of moderate strength. In Figure 15 the numerical solutions obtained at time 0.1 with both the WCD schemes for the nonconservative and the conservative formulations are compared. 


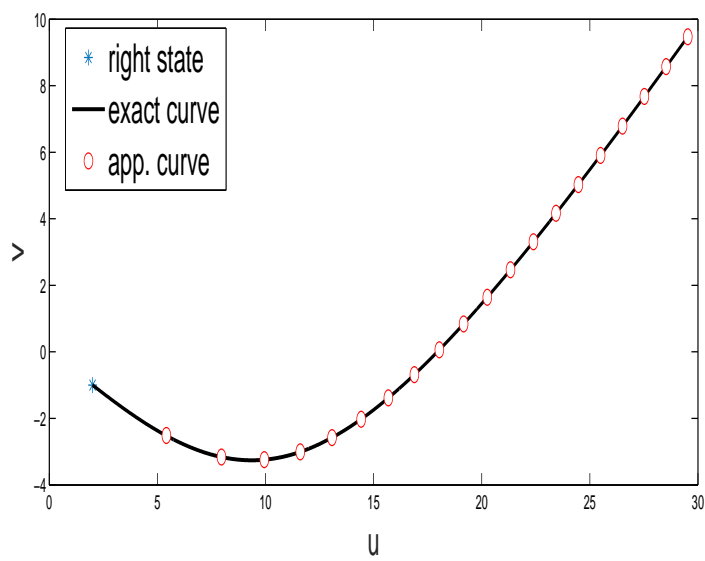

Fig. 9: Approximation of the Hugoniot curve of the right state $(2,-1)$ and the approximation obtained with $c=0.75, p=4$, and $\Delta x=0.004$.
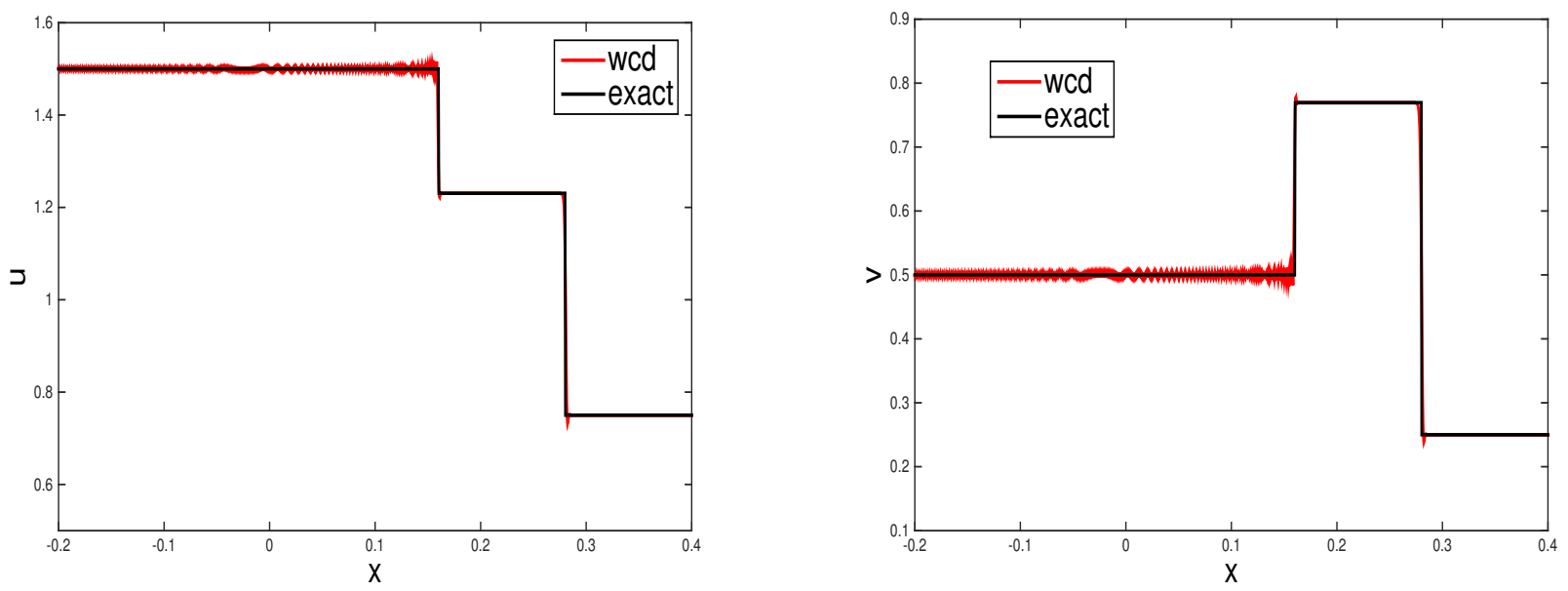

Fig. 10: Numerical results of the WCD method with $d x=1 / 2500, \tau=10^{-1}, p=4$ for the non-convex coupled cubic system with initial conditions (4.3) : Left: $u$, Right: $v$.

Next, we consider $h_{L}=3$ and $h_{R}=0.5$ to realize shocks of larger amplitude. In Figure 16, we present the numerical solutions obtained with both approaches. It can be seen that in the two considered cases the numerical solutions obtained with both approaches are in excellent agreement.

\subsection{The two-layer shallow water model}

Finally, we consider the two-layer shallow water system (2.19) with parameters $g=9.8$ and $r=0.98$. We consider the Riemann problem proposed in [10] with data

$$
U_{l}=\left[\begin{array}{l}
1.8 \\
0 \\
0.2 \\
0
\end{array}\right], \quad U_{r}=\left[\begin{array}{l}
0.2 \\
0 \\
1.8 \\
0
\end{array}\right] .
$$

In order to apply the WCD methods, we consider the regularization (2.21). 

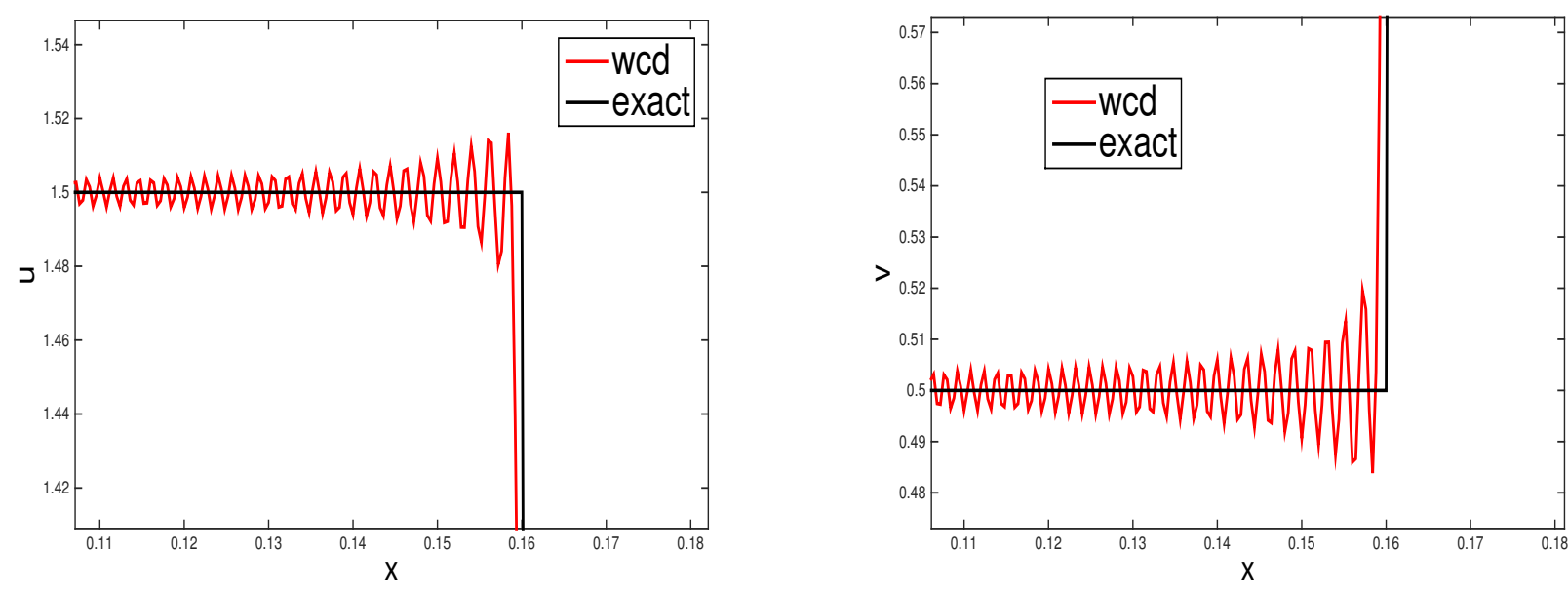

Fig. 11: Numerical results of the WCD method with $d x=1 / 2500, \tau=10^{-1}, p=4$ for the non-convex coupled cubic system with initial conditions (4.3) : zoom. Left: $u$, Right: $v$.
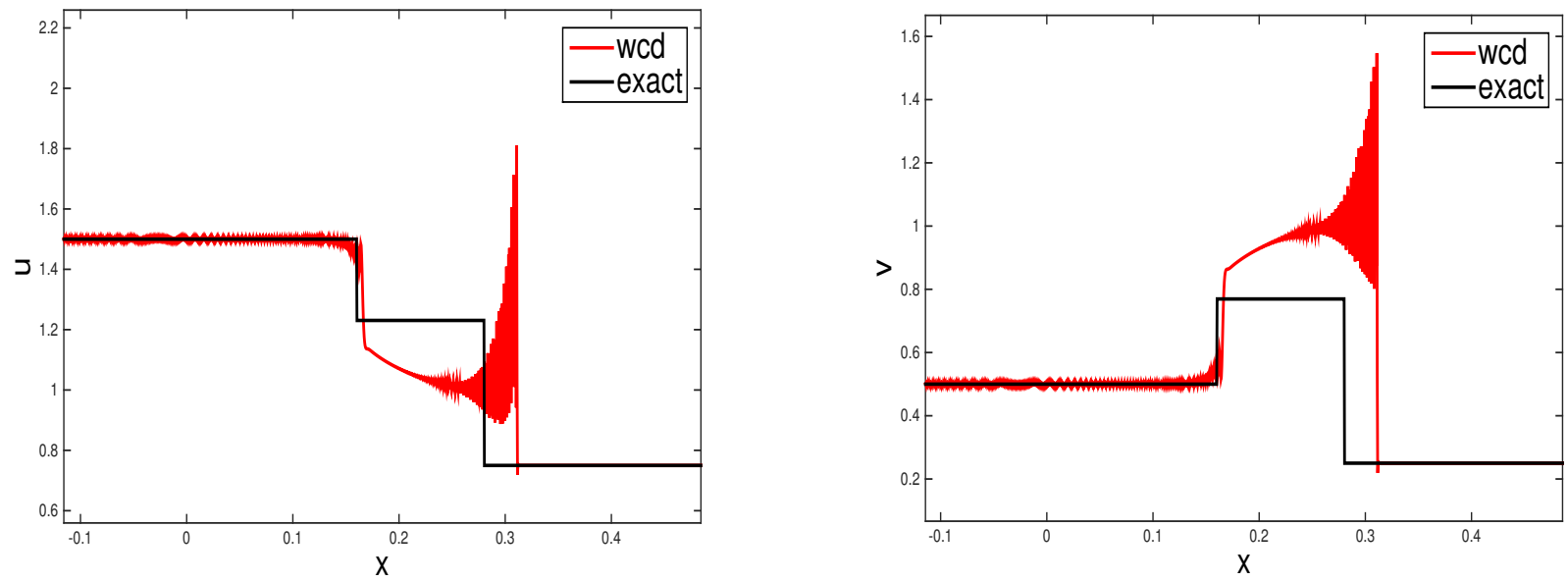

Fig. 12: Numerical results of the WCD method with $d x=1 / 2500, c=0.1, p=4$ for the coupled cubic system with initial conditions (4.3). Left: $u$, Right: $v$.

We consider first $\mu=1$ and apply the method with decreasing values of the tolerance to check the convergence of the method. The solution of the Riemann problem consists of two fast waves corresponding to the barotropic mode and tow compound slower internal waves (shock + rarefaction). Figure 17 shows the internal waves (variable $h_{2}$ ) at time $T=0.2$ together with a zoom of intermediate states captured by the numerical method depending on the value of $\tau$.

In order to study the sensitivity of the numerical solutions to changes in the viscous terms, we compare the numerical solutions obtained for different values of $\mu$ using the same value of $\Delta x$ and $\tau$. We also plot the numerical solutions obtained with the ICFP method introduced in [18], a path-conservative numerical method consistent with the family of straight segments. Figure 18 shows the numerical solutions and a zoom of the rarefaction part of the right compound internal wave.

Notice that the numerical solution obtained with the IFCP method is close to the numerical 


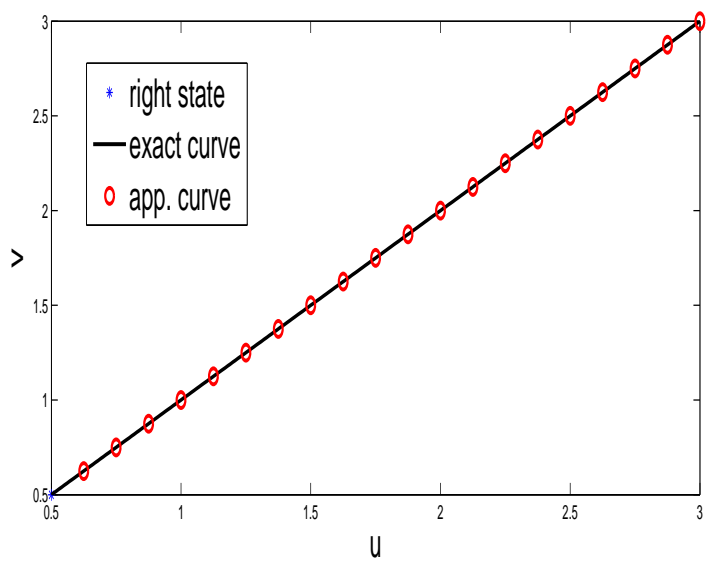

Fig. 13: Approximation of the Hugoniot curve of the right state $(.5, .5)$ for the cubic coupled system and the approximation obtained with $\tau=0.1, p=4$, and $\Delta x=1 / 1500)$.

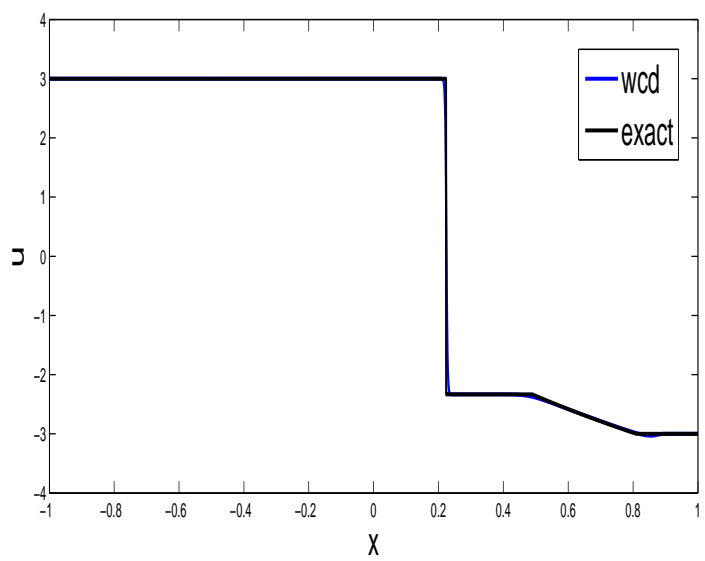

Fig. 14: Numerical results at $t=0.03$ of the WCD method for the Riemann problem (4.4) with $\Delta x=10^{-3}, \tau=10^{-1}$, and $p=6$.

solution obtained with $\mu=1$ but its distance to the WCD solution increases as $\mu$ decreases: remember that, in the viscous terms coming from the physics of the problem, $\mu=0$. This is very likely due to the fact that both the numerical viscosity matrix of the IFCP method and the diffusion matrix corresponding to the case $\mu=1$ commute with the matrix system $A(U)$ what makes that the corresponding traveling waves are close to each other: see [2].

The numerical method with $\mu=0$ becomes unstable: in this case, the diffusion matrix has a double null eigenvalue and thus the right-hand side of the stability condition (3.43) vanishes.

\section{Concluding remarks}

We have addressed the highly challenging problem of designing converging and accurate approximation methods for nonlinear hyperbolic systems in nonconservative form. The weak solutions under consideration are understood in the sense of Dal Maso, LeFloch and Murat [15] and defined via a family of Lipschitz continuous paths, especially traveling wave trajectories, as advocated in LeFloch [26]. Indeed, small-scale effects are encoded by choosing a suitable family of paths, which are parametrized in terms of viscous profiles of an underlying diffusive (or diffusive-dispersive) 

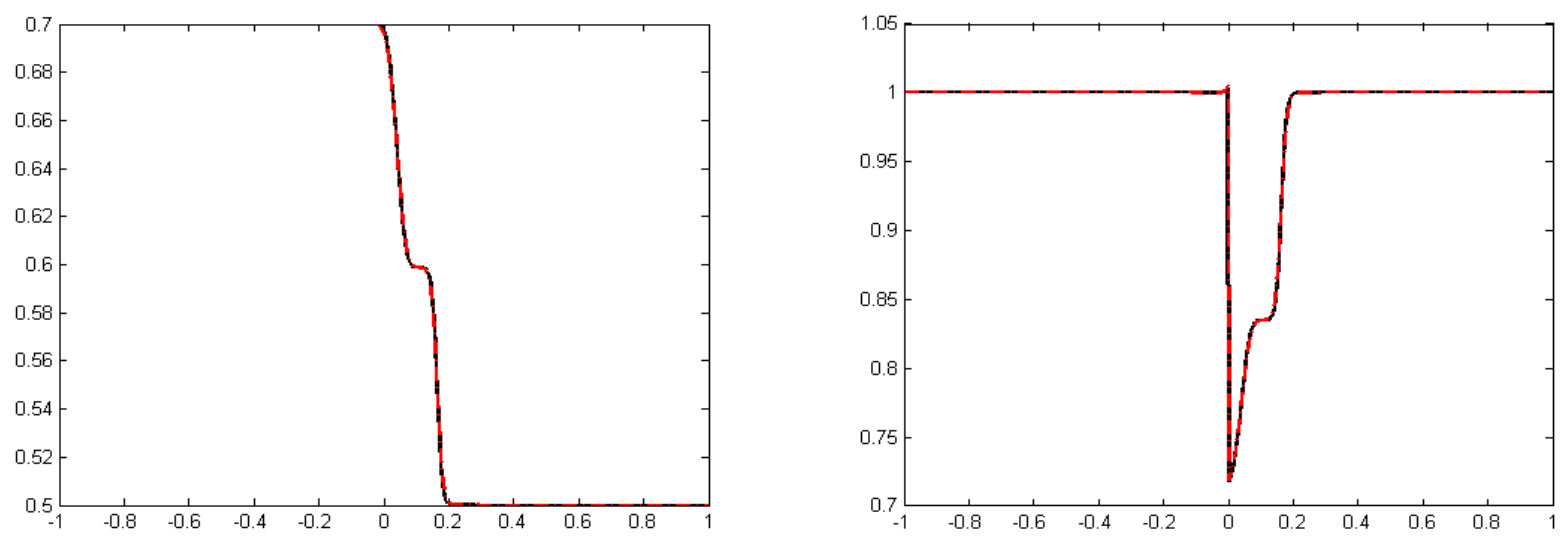

Fig. 15: Solution with a small shock for the modified shallow water system at time 0.1, using WCD schemes with $\tau=0.01, p=12, \Delta x=1 / 500$, and $C F L=0.4$. Left column: $h$. Right column: $u$. Black lines: solutions using the WCD scheme for the nonconservative system. Red lines: solutions using the WCD scheme for conservative systems.

regularization of the nonconservative hyperbolic system under consideration. The correct choice of the family of paths is given by traveling waves of the regularized system. Different choices of regularizations (or paths) usually lead to different weak solutions. Hence, the construction of accurate numerical schemes is an essential and particularly involved task, since high-order error terms need to be controlled in order to capture the underlying small-scale effects.

In the present paper, we have constructed accurate and robust finite difference schemes for nonconservative hyperbolic systems by adapting LeFloch-Mishra's WCD framework [32]. An analysis of the equivalent equation associated with the scheme (near a discontinuity) provides us with the basic tool in our construction. We have introduced arbitrarily high-order and consistent schemes containing a time-dependent parameter, which we have chosen so that the high-order terms of the equivalent equation are controlled by (and negligible with respect to) the low-order diffusive(dispersive) regularization terms. We have clearly demonstrated that such a choice of parameters is possible and we have derived conditions on the time-step in order to ensure linear stability.

The resulting WCD schemes were tested on four examples of nonconservative hyperbolic systems. Our numerical results illustrate the viability of our approach and demonstrate that the WCD schemes approximate the correct small-scale dependent shock waves, including shock waves of large amplitude, understood in the DLM sense.

The application of these schemes to other realistic models such as multi-phase flows would be a natural extension of our framework. Furthermore, while our presentation focused on nonconservative hyperbolic systems in a single space dimension, it is expected that the proposed WCD schemes should generalize to multi-dimensional problems. It would also be interesting to carry out a deeper analysis of the stability condition.

\section{Acknowledgments}

The authors gratefully acknowledge Manuel J. Castro for providing them with the numerical solution of the IFCP method applied to the two-layer shallow water system, and an anonymous referee for his/her detailed and helpful comments. This paper was completed in April 2015 when the second author (PLF) enjoyed the hospitality of the Courant Institute of Mathematical Sciences at New York University. The second author (PLF) was also partially supported by the Agence Nationale de la Recherche through the grant ANR SIMI-1-003-01. The fourth author (CP) was 

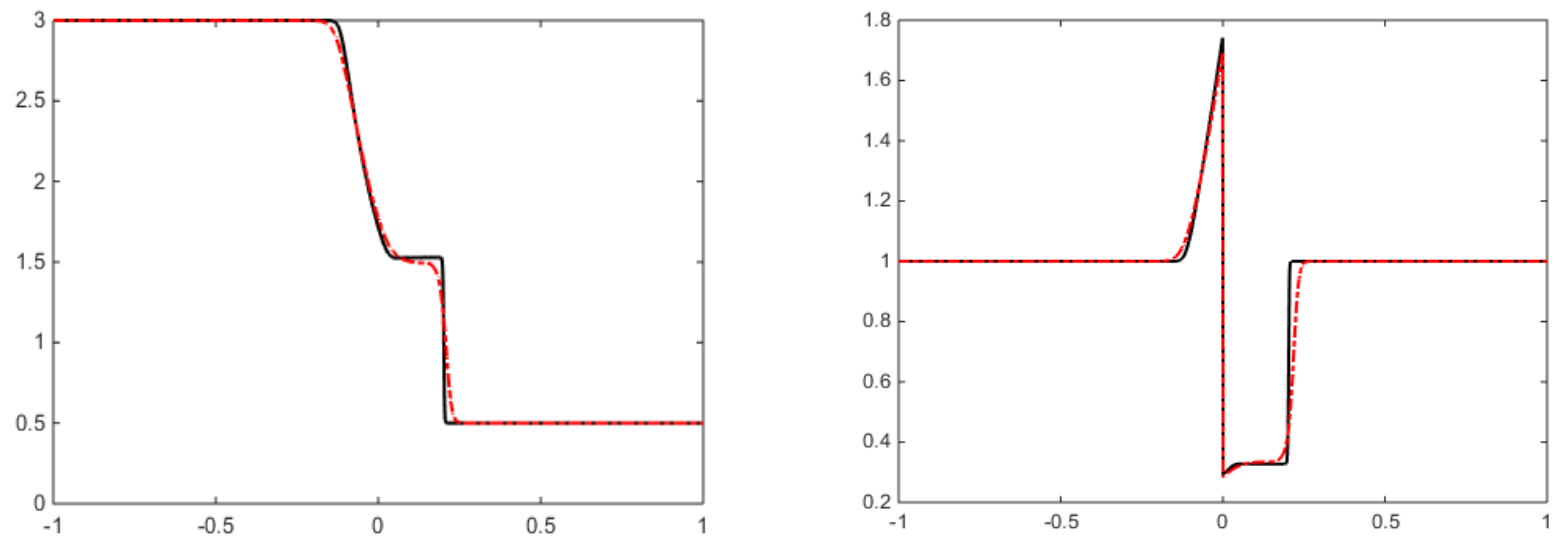

Fig. 16: Solution with a large shock for the modified shallow water system at time 0.1 using WCD schemes with $\tau=0.01, p=12, \Delta x=1 / 500$, and $C F L=0.31$. Left column: $h$. Right column: $u$. Black lines: solutions using the WCD scheme for the nonconservative system. Red lines: solutions using the WCD scheme for conservative systems.

partially supported by the Spanish Government and by FEDER through the Research project MTM2015-70490-C2-1-R, as well as by the Andalusian Government through the project P11FQM8179.

\section{References}

[1] R. Abgrall and S. Karni, A comment on the computation of nonconservative products, J. Comp. Phys. 229 (2010), 2759-2763.

[2] F. Allouges and B. Merlet. Approximate shock curves for non-conservative hyperbolic systems in one space dimension. J. Hyp. Diff. Eq. 1 (2004), 769-788.

[3] C. Berthon, Nonlinear scheme for approximating a nonconservative hyperbolic system, C. R. Math. Acad. Sci. Paris 335 (2002), 1069-1072.

[4] C. Berthon, B. Boutin, and R. Turpault, Shock profiles for the shallow-water Exner models, Adv. Applied Math. Mech. (2016), to appear.

[5] C. Berthon and F. Coquel, Nonlinear projection methods for multi-entropies Navier-Stokes systems. In "Finite Volumes for Complex Applications II: Problems and Perspectives", Hermes Science Publ., 1999, pp. 307-314.

[6] C. Berthon and F. Coquel, Nonlinear projection methods for multi-entropies Navier-Stokes systems, Math. Comp. 76 (2007), 1163-1194.

[7] C. Berthon, F. Coquel, and P.G. LeFloch, Why many theories of shock waves are necessary: Kinetic relations for nonconservative systems, Proc. Royal Soc. Edinburgh 137 (2012), 1-37.

[8] M. J. Castro, P.G. LeFloch, M.L. Muñoz-Ruiz, and C. Parés, Why many theories of shock waves are necessary: Convergence error in formally path-consistent schemes, J. Comput. Phys. 227 (2008), 8107-8129.

[9] M.J. Castro, U.S. Fjordholm, S. Mishra, and C. Parés, Entropy conservative and entropy stable schemes for nonconservative hyperbolic systems, SIAM J. Numer. Anal. 51 (2013), 1371-1391. 

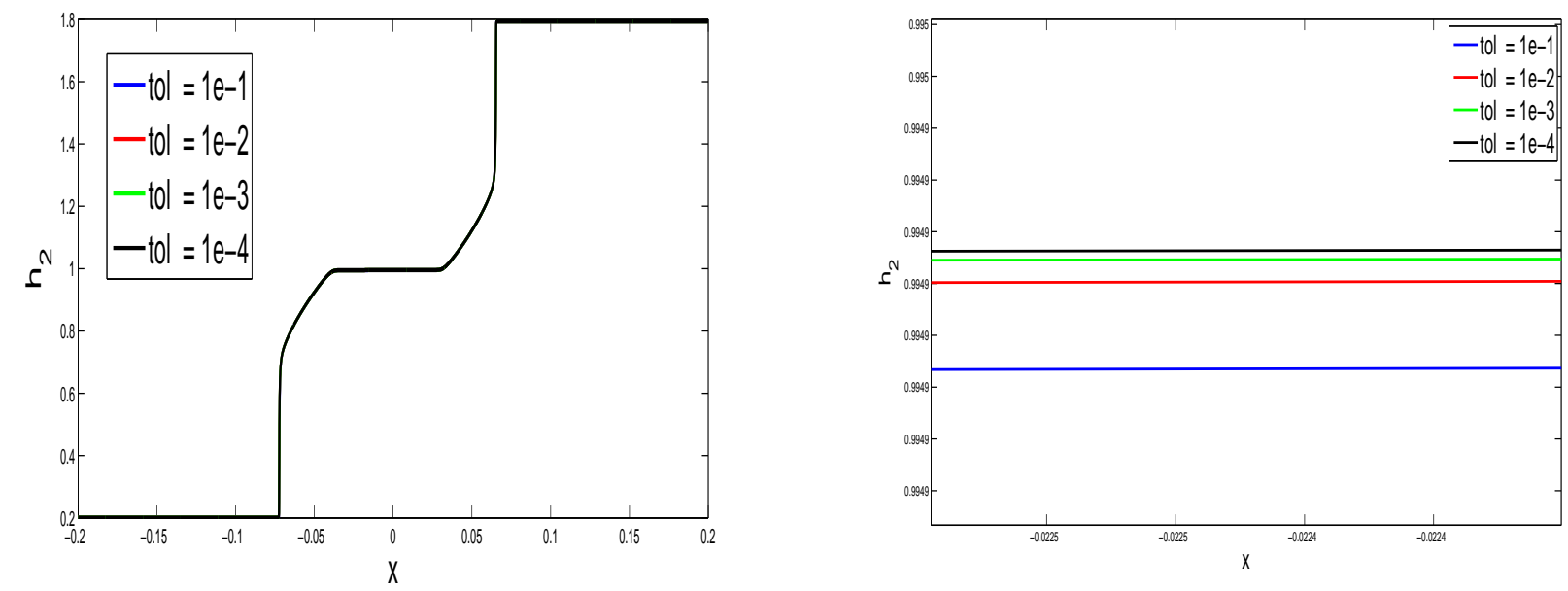

Fig. 17: Numerical results of the WCD method with $d x=1 / 12500$ and different values of $\tau$ for the two layer shallow water system with initial conditions (4.7) $: h_{2}$. Right: zoom of the intermediate state

[10] M.J. Castro, J.M. Gallardo, and C. Parés. High order finite volume schemes based on reconstruction of states for solving hyperbolic systems with nonconservative products. Applications to ShallowWater systems. Math. Comp. 75 (2006), 1103-1134.

[11] M.J Castro, J. Macías, and C. Parés, A Q-scheme for a class of systems of coupled conservation laws with source term: Application to a two-layer 1-D shallow water system, M2AN: Math. Model. Numer. Anal. 35 (2001), 107-127.

[12] M.J. Castro, C. Parés, G. Puppo, and G. Russo, Central schemes for nonconservative hyperbolic systems, SIAM J. Sci. Comput. 34 (2012), 523-558.

[13] C. Chalons and F. Coquel, Numerical capture of shock solutions of nonconservative hyperbolic systems via kinetic functions. In Analysis and Simulation of Fluid Dynamics, Advances in Mathematical Fluid Mechanics, Birkhäuser, 2007, pp. 45-68.

[14] C. Chalons and P.G. LeFloch, High-order entropy conservative schemes and kinetic relations for van der Waals fluids, J. Comput. Phys. 167 (2001), 1-23.

[15] G. Dal Maso, P.G. LeFloch, and F. Murat, Definition and weak stability of nonconservative products, J. Math. Pures Appl. 74 (1995), 483-548.

[16] M. Dumbser, M.J. Castro, C. Parés, and E.F. Toro, ADER schemes on unstructured meshes for nonconservative hyperbolic systems: Applications to geophysical flows, Comp. \& Fluids 38 (2009), $1731-1748$.

[17] J. Ernest, P.G. LeFloch, and S. Mishra, Schemes with well-controlled dissipation, SIAM J. Numer. Anal. 53 (2015), 674-699.

[18] E.D. Fernández-Nieto, M.J. Castro, and C. Parés. On an intermediate field capturing Riemann solver based on a parabolic viscosity matrix for the two-layer shallow water system. J. Sci. Comp. 48 (2011), 117-140.

[19] U. S. Fjordholm and S. Mishra, Accurate numerical discretizations of nonconservative hyperbolic systems, M2AN: Math. Model. Numer. Anal. 46 (2012), 187-296.

[20] L. Gosse, A well-balanced scheme using nonconservative products designed for hyperbolic systems of conservation laws with source terms, Math. Mod. Meth. Appl. Sci. 11 (2001), 339-365. 

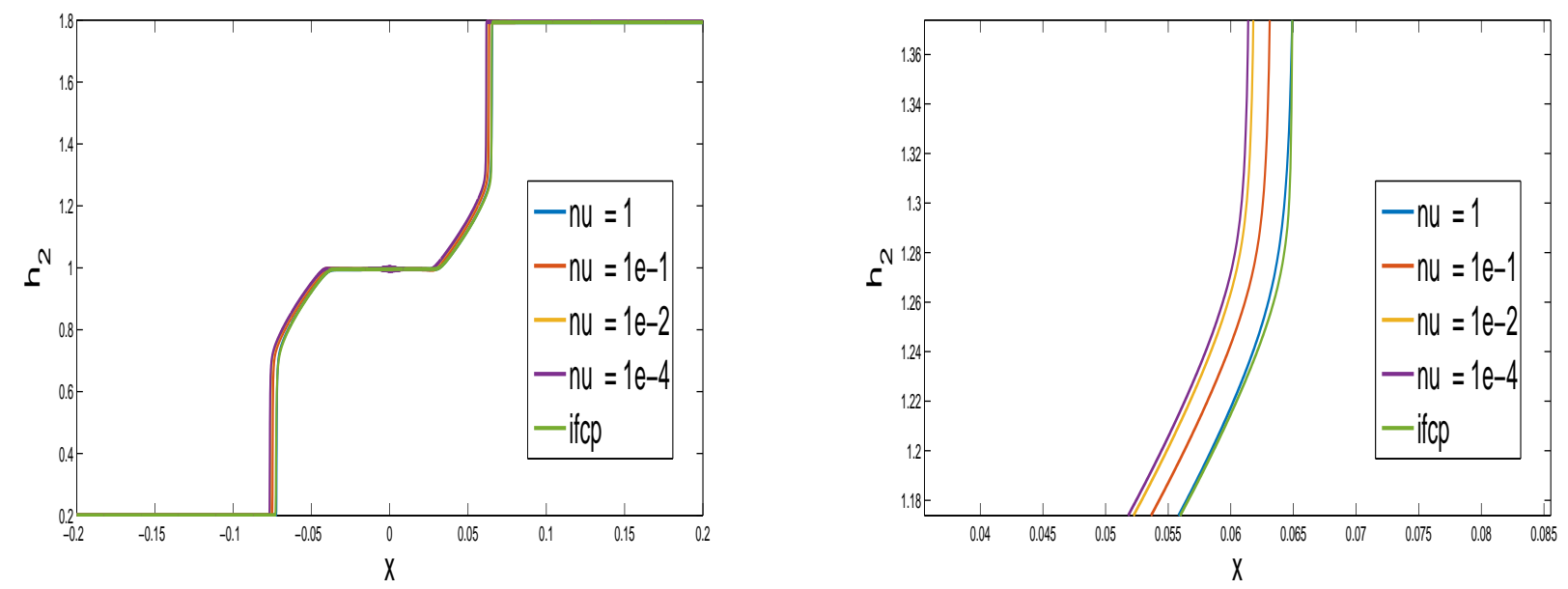

Fig. 18: Numerical results of the WCD method with $d x=1 / 12500, \tau=10^{-3}$ and different values of $\mu$ for the two layer shallow water system with initial conditions (4.7) $: h_{2}$. Right: zoom.

[21] B.T. Hayes and P.G. LeFloch, Nonclassical shocks and kinetic relations: Finite difference schemes, SIAM J. Numer. Anal. 35 (1998), 2169-2194.

[22] T.Y. Hou and P.G. LeFloch, Why nonconservative schemes converge to wrong solutions: Error analysis, Math. Comp. 62 (1994), 497-530.

[23] T.Y. Hou, P. Rosakis, and P.G. LeFloch, A level set approach to the computation of twinning and phase transition dynamics, J. Comput. Phys. 150 (1999), 302-331.

[24] S. Karni. Viscous shock profiles and primitive formulations, SIAM J. Num. Anal. 29 (1992), 15921609.

[25] P.G. LeFloch, Entropy weak solutions to nonlinear hyperbolic systems in nonconservative form, Comm. Part. Diff. Equ. 13 (1988), 669-727.

[26] P.G. LeFloch, Shock waves for nonlinear hyperbolic systems in nonconservative form, Institute for Math. and its Appl., Minneapolis, Preprint \# 593, 1989. Available at: https://conservancy.umn.edu/bitstream/handle/11299/5107/593.pdf

[27] P.G. LeFloch, On some nonlinear hyperbolic problems, Memoir of "Habilitation à Diriger des Recherches", Université Pierre et Marie Curie, Paris, July 1990.

[28] P.G. LeFloch, Propagating phase boundaries: Formulation of the problem and existence via the Glimm scheme, Arch. Ration. Mech. Anal. 123 (1993), 153-197.

[29] P.G. LeFloch, Hyperbolic Systems of Conservation Laws. The theory of classical and nonclassical shock waves, Lectures in Mathematics, ETH Zürich, Birkhäuser, 2002.

[30] P.G. LeFloch, Kinetic relations for undercompressive shock waves: Physical, mathematical, and numerical issues. In Nonlinear Partial Differential Equations and Hyperbolic Wave Phenomena, Vol. 526 of Contemporary Mathematics, AMS (2010) 237-272.

[31] P.G. LeFloch and T.-P. Liu, Existence theory for nonlinear hyperbolic systems in nonconservative form, Forum Math. 5 (1993), 261-280.

[32] P.G. LeFloch and S. Mishra. Numerical methods with controlled dissipation for small-scale dependent shocks. Acta Num. 23 (2014), 743-816.

[33] P.G. LeFloch and M. Mohamadian, Why many shock wave theories are necessary. Fourth-order models, kinetic functions, and equivalent equations, J. Comput. Phys. 227 (2008), 4162-4189. 
[34] P.G. LeFloch and C. Rohde, High-order schemes, entropy inequalities, and nonclassical shocks, SIAM J. Numer. Anal. 37 (2000), 2023-2060.

[35] C. Parés, Numerical methods for nonconservative hyperbolic systems: a theoretical framework, SIAM J. Num. Anal. 44 (2006), 300-321.

[36] C. Parés, Path-conservative numerical methods for nonconservative hyperbolic systems, In Numerical methods for balance laws, Vol. 24, Quaderni di matematica, Dipto. di Matematica della Seconda Universitá di Napoli, 2009, pp. 67-122.

[37] C. Parés and M.L. Muñoz. On some difficulties of the numerical approximation of nonconservative hyperbolic systems, Bol. Soc. Esp. Mat. Apl. 47 (2009), 23-52.

[38] L. Sainsaulieu and P.-A. Raviart, A nonconservative hyperbolic system modeling spray dynamics: solution of the Riemann problem, Math. Models Methods Appl. Sci. 5 (1995), 297-333.

[39] C.-W. Shu and S. Osher. Efficient implementation of essentially non-oscillatory shock-capturing schemes. J. Comp. Phys. 77 (1988), 439-471.

[40] H. B. Stewart and B. Wendroff, Two-phase flow: models and methods, J. Comput. Phys. 56 (1984), 363-409.

[41] G.B. Whitham. Linear and Nonlinear Waves. John Wiley and Sons, 2011. 